Rabaska

Revue d'ethnologie de l'Amérique française

\title{
L'inventaire du patrimoine immatériel religieux du Québec : bilan et perspectives
}

\section{Laurier Turgeon}

Volume 13, 2015

Présence de Marius Barbeau : l'invention du terrain en Amérique

française. Autour d’un legs centenaire (1914-2014)

URI : https://id.erudit.org/iderudit/1033773ar

DOI : https://doi.org/10.7202/1033773ar

Aller au sommaire du numéro

Éditeur(s)

Société québécoise d'ethnologie

ISSN

1703-7433 (imprimé)

1916-7350 (numérique)

Découvrir la revue

Citer cet article

Turgeon, L. (2015). L'inventaire du patrimoine immatériel religieux du Québec : bilan et perspectives. Rabaska, 13, 325-371.

https://doi.org/10.7202/1033773ar d'utilisation que vous pouvez consulter en ligne. 


\section{L'inventaire du patrimoine immatériel religieux du Québec : bilan et perspectives ${ }^{1}$}

LAURIER TURGEON

Chaire de recherche du Canada en patrimoine ethnologique Université Laval

Je dédie cet article à Marius Barbeau en hommage à sa contribution magistrale au patrimoine culturel immatériel du Québec.

\section{Introduction}

Cet article s'inscrit dans la continuité de l'œuvre pionnière et magistrale de Marius Barbeau dans le domaine du patrimoine immatériel religieux du Québec. Lors de sa riche et longue carrière de chercheur et de professeur, Marius Barbeau avait identifié et étudié tous les champs de ce que nous appelons aujourd'hui le patrimoine culturel immatériel - les traditions et expressions orales, les arts du spectacle, les pratiques sociales, rituels et événements festifs, les connaissances et pratiques concernant la nature et l'univers, les savoir-faire liés à l'artisanat traditionnel - des francophones et aussi de plusieurs groupes amérindiens de l'Amérique du Nord. Plus encore, il a posé les bases de l'étude du domaine du religieux en tant que patrimoine, notamment par son livre Saintes Artisanes, publié en 1943-1944². En effet,

1. Je tiens d'abord à remercier chaleureusement les fonctionnaires du ministère de la Culture et des communication du Québec pour avoir lancé ce projet et pour leur confiance, notamment Gérald Grandmont, Fernand Lévesque, Bernard Genest, Daniel Lauzon et René Bouchard. Je remercie ensuite $\mathrm{du}$ fond du cœur tous les partenaires du projet, qui ont toujours fait preuve d'une collaboration exemplaire, à commencer par Mission patrimoine religieux, tous les membres du comité de consultation dont la liste se trouve ci-dessous, la cinquantaine de communautés religieuses qui ont participé directement à l'inventaire et les nombreux informatrices et informateurs religieux qui ont accepté de transmettre des éléments de leur patrimoine immatériel. Mes remerciements vont aussi à tous les collaborateurs - professionnels, postdoctorants et étudiants - de la Chaire de recherche du Canada en patrimoine ethnologique dont la très longue liste se trouve sur le site de l'inventaire (www.ipir.ulaval.ca). Ma gratitude est grande envers mon collègue Jean Simard qui m'a initié au patrimoine religieux et qui a commenté une première version de cet article. Qu'il trouve ici l'expression de ma profonde reconnaissance.

2. L'ouvrage est publié en deux volumes à un an d'intervalle : Marius Barbeau, Saintes Arti- 
il a bien démontré que de nombreux métiers artisanaux et artistiques sont pratiqués dans les communautés religieuses féminines du Québec, tant la broderie et la peinture que la sculpture, la dorure, la reliure, le tissage, la dentellerie, la pâtisserie et la confiserie. Comme l'indique le titre de son livre, en combinant un terme (saintes) qui renvoie au sacré et un autre (artisanes) qui renvoie au patrimoine, Marius Barbeau établit un rapprochement entre ces deux domaines et révèle que le monde religieux n'est pas seulement sacré et cultuel, mais aussi culturel et patrimonial. Par la même occasion, il nous livre un des premiers inventaires détaillés des collections et des savoir-faire artistiques et artisanaux des anciennes communautés religieuses du Québec.

En souhaitant poursuivre et contribuer à l'œuvre de Marius Barbeau, dans cet article nous voulons présenter un bilan critique et des perspectives de développement de l'Inventaire du patrimoine immatériel religieux du Québec (IPIR : WwW.IPIR.ULAVAL.CA). La réalisation de cet inventaire est née d'une préoccupation de la part du gouvernement et de la société civile pour la sauvegarde du patrimoine immatériel religieux (les traditions orales, les savoir-faire artistiques et artisanaux, les rituels, les fêtes, les pratiques concernant la nature et l'univers) jugé, de tous les patrimoines religieux (matériel, artistique, immobilier, mobilier), le moins bien protégé et le plus fragile. $\mathrm{Au}$ Québec, jusqu'en 2005-2006, on avait inventorié et protégé surtout le patrimoine religieux matériel, peu le patrimoine immatériel. Par exemple, l'entente signée entre le ministère de la Culture et des communications (MCCQ) et la Fondation du patrimoine religieux en 1996 précise que l'inventaire doit porter sur les immeubles, les meubles et les archives. Nulle part il n'est fait mention du domaine de l'immatériel. Véritable pionnier dans ce domaine, Jean Simard avait dès la fin des années 1990 bien circonscrit toute la portée de ce patrimoine dans les communautés religieuses et son caractère à la fois très riche et menacé ${ }^{3}$. Il avait dès lors identifié l'importance de réaliser un inventaire des traditions orales des communautés religieuses. Dans son sillage, Diane Audy mènera un inventaire du patrimoine immatériel dans les trois couvents d'augustines de Québec avec le soutien financier du ministère de la Culture et des communications du Québec ${ }^{4}$. Un autre moment fort de ce mouvement

sanes I. Les brodeuses, Montréal, Fides, 1943 ; et Saintes Artisanes II. Mille petites adresses, Montréal, Fides, 1944.

3. Jean Simard, avec la collaboration de Nicole Bourgault et al., « Le Patrimoine immatériel des augustines de 1'Hôtel-Dieu de Québec ». Rapport d'enquête orale préparé sous la direction de Jean Simard, assisté de David Harvengt, Québec, Université Laval, mai 1997, 183 p. De ce rapport fut tiré un article du même auteur, "Le Patrimoine immatériel des communautés religieuses ", Les Cahiers des Dix, $\mathrm{n}^{\circ}$ 53, 1999, p. 251-283.Voir également Jean Simard, Le Patrimoine religieux au Québec. Exposé de la situation et orientations, Québec, Gouvernement du Québec, Commission des Biens culturels, 1998, $55 \mathrm{p}$.

4. Diane Audy, «Enquête orale auprès des Augustines de la Miséricorde de Jésus des monastères de l'Hôtel-Dieu de Québec, l'Hôpital Général de Québec, l'Hôtel-Dieu du Sacré-Cœur de Jésus de 
de sensibilisation du gouvernement et de la société civile à l'important enjeu que représentait le patrimoine immatériel religieux du Québec fut le colloque international de novembre 2004 tenu à l'église Saint-Dominique à Québec, à deux pas du MccQ, qui a mobilisé plus de 650 personnes de tous les milieux et de toutes les régions du Québec ${ }^{5}$.

Dans les mois qui ont suivi, le gouvernement du Québec a décidé de mettre sur pied une commission parlementaire et de consulter la population québécoise pour faire le point et envisager un programme d'interventions spécifiquement sur le patrimoine religieux du Québec. Le rapport de la Commission, déposé en juin 2006, attirait l'attention sur l'importance de préserver le patrimoine immatériel religieux, en faisant remarquer, entre autres, que c'est souvent le patrimoine immatériel qui donne sens au patrimoine matériel. Ce sont les traditions orales, les savoir-faire, les fêtes, les rites et la mémoire qui permettent de comprendre les bâtiments et les objets matériels et de leur donner vie. Le rapport soulignait aussi que le patrimoine immatériel était de tous les patrimoines religieux celui qui était le plus menacé. Contrairement au patrimoine matériel qui est fait justement pour durer dans le temps et faire face aux épreuves du temps, le patrimoine immatériel est porté par des personnes et il demeure aussi fragile que la vie elle-même. En effet, ce sont des personnes qui le conservent et qui le transmettent. C'est donc une perte irréversible lorsqu'un porteur de traditions meurt. Or, le rapport faisait remarquer que les communautés religieuses sont vieillissantes (la moyenne d'âge dépassait 80 ans dans plusieurs communautés) et que la mort frappe souvent subitement et sans avertissement chez les personnes âgées ${ }^{6}$. À la lumière de ces constats, la Commission recommandait la réalisation d'enquêtes sur le patrimoine immatériel religieux. En effet, la recommandation 7 préconisait qu'un « programme d'enquêtes sur le patrimoine religieux immatériel soit coordonné dans les plus brefs délais [...] et que soit élaborée, en collaboration avec les établissements d'enseignement et les musées, avec la participation des communautés et des groupes qui créent, entretiennent et transmettent ce

Québec », rapport préparé pour le Ministère de la Culture et des communications de Québec, 2004. Ces enregistrements oraux, conservés sur bande magnétique, ont été déposés au monastère des Augustines et au ministère de la Culture et des communications.

5. Les principales contributions de ce colloque ont été publiées sous la direction de Laurier Turgeon, Le Patrimoine religieux du Québec : entre le cultuel et le culturel, Québec, Presses de 1'Université Laval, 2005.

6. Par exemple, les Augustines au monastère de l'Hôtel-Dieu de Québec, fondatrices du premier hôpital en Nouvelle-France, qui comptaient 172 religieuses en 1970, ne sont plus en 2014 que 26, dont 13 sont à l'infirmerie. La moyenne d'âge de la communauté atteint plus de 80 ans. Cinq des six monastères en région (Gaspé, Montmagny, Saint-Georges, Lévis et Roberval) ont été fermés et leurs biens et personnes regroupés à la maison-mère et au monastère de l'Hôtel-Dieu de Québec. On pourrait multiplier les exemples. Information fournie par sœur Hélène Marquis, supérieure générale des Augustines du Québec (communication personnelle, avril 2014). 
patrimoine, une stratégie d'intervention cohérente et efficace dans le but de connaître, de protéger et de transmettre le patrimoine religieux immatériel du Québec ${ }^{7} »$.

C'est dans ce contexte que, dès l'automne 2006, la Chaire de recherche du Canada en patrimoine ethnologique formait, à la demande du Ministère, un comité de consultation réunissant des représentants des communautés religieuses, d'organismes voués à la sauvegarde et à la préservation du patrimoine religieux, des responsables du patrimoine religieux au Ministère, des experts et des responsables de la Chaire ${ }^{8}$. Établi par le Ministère, le mandat du comité était de développer un programme d'inventaire ethnologique du patrimoine immatériel religieux du Québec et de proposer une stratégie pour sa réalisation afin d'avoir un état des lieux de ce patrimoine, ce qui constituait une première étape nécessaire à toute construction de connaissances sur un sujet et d'envisager des mesures de sauvegarde.

Avant de procéder à sa mise en œuvre à l'échelle du Québec, il a été convenu de réaliser une phase de travaux préparatoires afin d'évaluer la nature et l'étendue du patrimoine immatériel religieux. Ce travail a pris la forme d'un projet pilote destiné à développer une méthodologie d'inventaire multimédia pour conserver le caractère vivant du patrimoine immatériel et le rendre facilement accessible aux communautés religieuses, aux spécialistes du patrimoine religieux et au grand public au moyen du Web. Il s'agissait d'un défi de taille, car jusqu'alors il n'existait nulle part au monde d'inventaire multimédia en ligne du patrimoine immatériel religieux aussi élaboré, tant sur le plan méthodologique que sur le plan technologique. Comme le patrimoine immatériel religieux est constitué essentiellement de pratiques traditionnelles exprimées par la voix et le geste, la captation audiovisuelle permettait de préserver le caractère vivant de ces pratiques et représentait donc un moyen privilégié de conservation, de mise en valeur, de transmission et de diffusion. Inspirée de l'expertise développée par la Chaire dans le cadre de l'Inventaire

7. «Croire au patrimoine religieux du Québec », rapport préparé par la Commission de la Culture, et publié par le Secrétariat des commissions de l'Assemblée nationale du Québec, juin 2006, p. 21.

8. Ce comité était composé notamment : du président de la Commission des biens culturels du Québec ; de la présidente de Mission patrimoine religieux ; de la supérieure générale des Augustines de la Miséricorde de Jésus ; du supérieur général du Séminaire de Québec ; de la directrice du Réseau InfoMuse de la Société des musées québécois ; d'un expert en patrimoine religieux de la Société québécoise d'ethnologie ; du chargé de mission en patrimoine religieux à la Direction générale des politiques, du patrimoine et du financement, ministère de la Culture, des communications et de la condition féminine du Québec (MсCF); de la conseillère en patrimoine à la Direction du patrimoine et de la muséologie, MccF ; du directeur du Conseil du patrimoine religieux du Québec ; du directeur du Musée des religions de Nicolet ; du directeur de la Corporation du patrimoine et du tourisme religieux de Québec ; de la directrice du Musée des Ursulines de Québec ; de la directrice de la Société du patrimoine religieux du diocèse de Saint-Hyacinthe ; de la coordonnatrice du projet d'inventaire à la Chaire de recherche du Canada en patrimoine ethnologique de l'Université Laval ; et du titulaire de la Chaire. 
des ressources ethnologiques du patrimoine immatériel du Québec (www. irepi.ulaval.ca), l'équipe de réalisation a cherché à adapter l'approche plus spécifiquement au patrimoine immatériel religieux, à peaufiner la méthodologie d'enquête de terrain, à améliorer les techniques de prélèvement et de traitement des données numériques et à rendre très efficace le fonctionnement du site destiné à transmettre et à diffuser les informations. En 2007-2008, la Chaire menait ce projet pilote auprès de neuf communautés de religieux et de fidèles de différentes confessions ( 4 catholiques, 2 protestantes, 1 orthodoxe, 1 juive et 1 amérindienne). Les objectifs du projet étaient de tester les méthodes et les outils d'enquête et de procéder au traitement numérique des données en vue de la diffusion sur le Web. La méthodologie et les outils d'enquête ainsi que le système de classification du patrimoine immatériel religieux, élaboré à partir de la grille des pratiques culturelles de Jean Du Berger ${ }^{9}$, ont été mis à l'épreuve sur le terrain et ces expériences concrètes ont permis de raffiner les approches et de faire les ajustements qui s'imposaient.

Compte tenu des résultats très concluants du projet pilote, en 2008 le Ministère proposait à la Chaire de soumettre une demande de subvention destinée à réaliser un inventaire national du patrimoine immatériel religieux. À la suite de plusieurs séances de travail avec le comité consultatif, composé des mêmes membres que pour le projet pilote, la Chaire s'est engagée à réaliser un projet visant à inventorier les éléments du patrimoine immatériel religieux de différentes traditions religieuses dans environ 50 communautés de religieux et de fidèles afin d'avoir un échantillon des pratiques et des savoir-faire avec, comme objectif, l'établissement d'environ 850 à 900 fiches d'inventaire ( $80 \%$ pour les catholiques, $20 \%$ pour les autres confessions). Inspiré par la même volonté de diffusion des connaissances que dans le projet pilote, l'équipe de la Chaire a décidé de mettre les fiches en ligne au fur et à mesure du déroulement du projet, plutôt que d'attendre sa fin. Cette diffusion immédiate des résultats de l'inventaire, par le biais d'un site Web, permettrait de faire connaître le patrimoine immatériel religieux au grand public et aux publics ciblés, notamment aux élèves inscrits au nouveau programme d'Éthique et culture religieuses des écoles secondaires du Québec. Ce travail d'inventaire, cofinancé par le MccQ (72\%) et la Chaire (28\%), s'est échelonné sur quatre ans, soit de 2008 à 2012.

\section{Un inventaire multimédia numérique et participatif}

Tout en nous inspirant de l'approche ethnographique classique, axée sur les entrevues semi-dirigées et l'observation directe, nous avons réalisé un inventaire du patrimoine immatériel religieux, novateur de trois manières. Premièrement, nous avons exploité quatre médias numériques (texte, photo,

9. Jean Du Berger, Grille des pratiques culturelles, Québec, Éditions du Septentrion, 1997. 
enregistrement sonore et vidéo) pour capter, conserver et transmettre le caractère vivant de ce patrimoine. Le patrimoine immatériel est d'abord un patrimoine vivant et nous avons communiqué toute sa vitalité, notamment par la voix et le geste. Deuxièmement, nous avons rendu ce patrimoine accessible au plus grand nombre de personnes en recourant à une diffusion à partir d'un site à contenu riche, convivial et interactif. Troisièmement, nous avons favorisé une approche participative, en intégrant autant que possible les membres des communautés religieuses à toutes les étapes de l'inventaire, depuis le choix des informateurs et la cueillette des données jusqu'à la diffusion des informations.

Si les inventaires n'ont pas toujours eu bonne presse parmi les ethnologues et les chercheurs en sciences humaines, leur utilité est indéniable. Certains leur reprochent de transformer des traditions vivantes en archives, de sélectionner et de hiérarchiser, puis de figer les pratiques traditionnelles en les conservant en copie unique érigée en modèle. D'autres soutiennent qu'ils freinent l'évolution des pratiques culturelles et enlèvent à la culture traditionnelle son dynamisme. D'autres encore déclarent qu'ils coûtent trop cher et privent les praticiens de subventions pour développer leur propre art. Ces critiques anachroniques reposent sur des pratiques d'inventorisation désuètes qui datent des années 1960 et 1970 et révèlent une ignorance des méthodologies d'inventorisation actuelles. Comme souligné dans l'article 12 de la Convention pour la sauvegarde du patrimoine culturel immatériel de 1'UNESCO de 2003, les inventaires sont indispensables, car ils sont à la base de toute stratégie de conservation, de tout plan de sauvegarde, de toute connaissance sur le patrimoine, de toute gestion du patrimoine et de toute démarche scientifique, et qu'ils constituent un outil de sensibilisation, d'éducation et de transmission extrêmement efficace. En effet, la constitution d'un corpus d'informations, donc d'un inventaire, est un préalable à la mise en œuvre d'outils de gestion, à toute recherche scientifique, plus encore, à toute acquisition de connaissances sur laquelle reposent la sensibilisation et la transmission. Même les praticiens (artisans, conteurs, musiciens, chanteurs) réalisent de petits inventaires de différentes pratiques observées de manière informelle lorsqu'ils constituent leurs répertoires. Selon Cécile Duvelle, la directrice de la Section du patrimoine culturel immatériel à l'UNESCO, les inventaires représentent un outil essentiel de la Convention pour la sauvegarde du patrimoine culturel immatériel, me faisant remarquer que l'article 12 est l'un des rares articles contraignants de la Convention ${ }^{10}$. En effet, tout État

10. «Pour assurer l'identification en vue de la sauvegarde, chaque État partie dresse, de façon adaptée à sa situation, un ou plusieurs inventaires du patrimoine culturel immatériel présent sur son territoire. ", Convention pour la sauvegarde du patrimoine culturel immatériel, Paris, UnESCO, 2003, article 12 . 
partie a l'obligation de dresser un ou des inventaires sur son territoire à titre de signataire de la Convention et aussi pour être admissible à la présentation de candidatures sur les listes (Liste représentative ou Liste de sauvegarde urgente $\left.{ }^{11}\right)$. Elle considère que les inventaires font un travail beaucoup plus important de sauvegarde, de transmission, de participation et de mobilisation sociale que les listes qui demeurent plus sélectives et restrictives ${ }^{12}$. Elle souligne que l'UNESCO offre des formations sur l'inventorisation du patrimoine immatériel depuis plus de deux ans dans le cadre de son programme de renforcement des capacités destiné surtout aux pays du Sud. Elle fait aussi remarquer que l'inventorisation n'est pas un simple exercice de recensement, mais une pratique de sauvegarde à part entière. D'ailleurs, le guide de l'UNESCO utilisé pour la formation l'indique clairement :

Il faut se rappeler qu'un inventaire n'est pas seulement une liste (préparatoire) d'éléments immatériels ; c'est aussi le résultat d'un processus de consultation et de discussion qui conduit parfois ou souvent à un processus de sauvegarde. Inventorier est toujours une opération en cours parce qu'il faut toujours ajouter de nouveaux éléments à la liste et la tenir à jour (ce qui suppose aussi qu'on en retranche les éléments disparus) $)^{13}$.

C'est moins l'inventaire lui-même que l'on doit remettre en cause que les manières traditionnelles de le faire. Animés par la théorie des survivances, les ethnologues d'il n'y a pas si longtemps recherchaient les récits ou les pratiques les plus authentiques et en faisaient des objets de contemplation. Le but n'était pas de transmettre les traditions et de les laisser évoluer, mais plutôt de les conserver et de les figer dans leurs formes jugées les plus pures. Il faut dire que l'inventaire ethnologique des années 1950-1970, réalisé sur support analogique (bandes magnétiques et films), exigeait des équipements lourds, de longs séjours sur le terrain, et des conditions de conservation particulières (salles à température et à humidité contrôlées) qui entraînaient des coûts élevés. Ainsi, les enregistrements sonores et audiovisuels étaient-ils généralement réalisés une seule fois, essentiellement dans une perspective de préservation, conservés en exemplaire unique dans un lieu hautement sécuritaire et fermé à clé. Les praticiens, à commencer par les informateurs eux-mêmes, avaient difficilement accès à ces enregistrements qui, en raison de leur caractère singulier, étaient érigés en références quasiment incontournables.

11. Directives opérationnelles pour la mise en cuvre de la Convention pour la sauvegarde du patrimoine culturel immatériel, Adoptées par l'Assemblée générale des États parties à la Convention à sa deuxième session (Siège de 1'Unesco, Paris, 16-19 juin 2008).

12. Entrevues avec Cécile Duvelle aux bureaux du siège de l'UnESCO à Paris le 27 mars 2012 et le 14 mai 2014.

13. Document pour l'atelier de renforcement des capacités : Inventaires, 25 janvier 2011 ; voir site de l'UNESCO : www.unesco.org/culture/ich/index, consulté le 21 mai 2014. 
Plus qu'un simple inventaire destiné à la conservation, notre objectif a été de réaliser une base de données multimédia virtuelle qui facilite la transmission, la mise en valeur, la sensibilisation et la diffusion du patrimoine immatériel religieux. La transmission n' assure pas juste la conservation des traditions, elle contribue à les transformer, à les dynamiser, à les adapter à des nouveaux besoins et à les renouveler en fonction de ces nouveaux usages sociaux. Par la même occasion, elle participe à la valorisation et à la reconnaissance de ceux qui les transmettent. Il ne s'agissait pas de promouvoir le culte, bien entendu, mais de faire connaître et reconnaître les pratiques traditionnelles - cultuelles et culturelles - et leurs artisans en tant que patrimoine dans un souci d'éducation du grand public, de sensibilisation, de transmission, et de mise en valeur ${ }^{14}$. L'usage d'équipements d'enregistrement électroniques (appareils photos et vidéo numériques haute définition), de bases de données multimédia (texte, photo, enregistrement sonore, vidéo) et des outils d'exploitation en ligne de ces bases de données ont contribué à révolutionner les pratiques de l'inventaire du patrimoine immatériel. Les nouvelles technologies de l'information facilitent non seulement la fabrication, l'accès et la gestion des inventaires, elles suscitent de nouvelles façons de concevoir et de réaliser l'inventaire lui-même. L'accès aux données par le Web permet des appropriations et des réappropriations multiples par une large gamme de personnes (communautés elles-mêmes, journalistes, muséologues, chercheurs, enseignants, élèves, citoyens) et favorise l'évolution des pratiques et la valorisation sociale des communautés qui en sont les détentrices. En effet, l'inventaire multimédia en ligne devient un outil dynamique de communication et de transmission patrimoniale puis de développement culturel et social.

Nous avons adopté une définition large du patrimoine immatériel religieux qui déborde le caractère strictement religieux et spirituel pour atteindre la vie culturelle et sociale des religieuses et des religieux. Il faut d'abord dire que limiter le patrimoine religieux à la seule sphère du sacré serait très restrictif et nous ferait manquer l'essentiel, qui est d'ordre culturel. Par exemple, les pratiques religieuses et spirituelles des communautés religieuses catholiques sont très semblables, régies à peu près par les mêmes rites imposés par la doctrine de l'Église catholique romaine. Un inventaire qui porterait exclusivement sur le patrimoine sacré serait donc assez redondant, du moins pour les catholiques qui représentent la grande majorité de la population québécoise. La spécificité du patrimoine religieux québécois réside dans son adaptation au territoire, dans son histoire, dans la vocation particulière de chacune des congrégations et dans la culture institutionnelle qui se développe dans les communautés. C'est sans doute pour cette raison que tous les spécialistes

14. Rapport final - Évaluation du travail normatif de l'UNESCO [...]. Convention de 2003, UNESCO, 2013, 5.1.1, n 138 . 
proposent une définition étendue du patrimoine immatériel religieux. Par exemple, la corporation Mission patrimoine religieux englobe dans sa définition les savoir-faire de la vie communautaire, professionnelle et artisanale :

Le patrimoine immatériel est celui qui fait découvrir les us et coutumes qui découlent de l'esprit et du charisme des fondateurs ainsi que de la mission propre à chaque communauté ; des savoirs et des savoir-faire qui ont été développés et transmis de génération en génération dans chacune des institutions et qui se rapportent à la vie communautaire, à la vie religieuse, à la vie professionnelle ainsi qu'à l'artisanat pratiqué dans chacune des communautés ${ }^{15}$.

De même, Jean Simard, s'inspirant de la définition du patrimoine donnée en 2000 par le Groupe-conseil sur la politique du patrimoine culturel du Québec présidé par Roland Arpin, estime qu'est patrimoine tout ce qui est approprié collectivement pour sa valeur de témoignage et de mémoire historique : « En font partie des objets matériels mais aussi des savoirs et des savoirfaire immatériels que détiennent des porteurs de traditions ${ }^{16} »$. Il faut dire que ce sont ces savoir-faire et ces mémoires spécifiques qui représentent les éléments les plus menacés et les plus significatifs de ce patrimoine religieux avec la disparition prochaine de bon nombre de communautés religieuses. Avec cette préoccupation à l'esprit, nous avons ouvert l'enquête à un nombre assez grand de possibilités pour éviter de passer à côté de traditions uniques. Plutôt que d'imposer des choix "d'en haut », basés sur les seuls critères d'esthétisme, d'ancienneté ou d'exclusivité, nous avons invité les membres des communautés à choisir « par le bas » les éléments qui semblaient significatifs et valorisants pour elles.

Pour concrétiser la participation, la première mesure prise a été la constitution d'un comité de suivi composé de 13 membres, dont des membres des communautés religieuses (4), des représentants du milieu muséal (3), des fonctionnaires du MCcQ (2), des représentants du milieu associatif (2) et des spécialistes universitaires du patrimoine religieux (2). Établi par le Ministère, le mandat du comité a été d'assurer le suivi et le bon fonctionnement du programme d'inventaire ethnologique du patrimoine immatériel religieux du Québec. Les représentants des communautés religieuses ont pris une part très active au développement d'une stratégie d'inventorisation, à l'identification des communautés et des éléments à inventorier et à l'établissement de contacts avec les religieux et les religieuses. La deuxième mesure a consisté en l'élaboration d'un partenariat avec les communautés religieuses. Le partenaire principal de la Chaire pour l'inventaire a été Mission patrimoine religieux (MPR) qui regroupe une centaine de religieux et de laïcs provenant de toutes

15 Bulletin de Mission patrimoine religieux, $\mathrm{n}^{\circ}$ 8, novembre 2005, p. 10.

16. Jean Simard, Yves Bergeron et Marcel Masse, « Mémoire sur l'avenir du patrimoine religieux » présenté à la Commission de la culture de l'Assemblée nationale du Québec, janvier 2006. 
les régions du Québec dédiés à la mise en valeur du patrimoine religieux. Par le biais d'une concertation avec l'ensemble des communautés religieuses de la province, MPR a recensé, répertorié et rendu accessible le patrimoine des communautés religieuses. Michelle Paradis, ethnologue et muséologue, et par la suite Marie-Thérèse Bournival, également muséologue, ont été chargées par MPR de rencontrer les communautés religieuses et d'identifier avec elles les éléments du patrimoine immatériel à inventorier. Au cours du projet, le partenariat s'est étendu au-delà des communautés religieuses. La Chaire a collaboré avec la Société des musées québécois, qui avait mandat de faire l'inventaire du patrimoine mobilier des communautés religieuses, pour étudier la mise en œuvre de l'intégration des éléments du patrimoine mobilier et du patrimoine immatériel religieux des inventaires. Cette concertation entre les deux partenaires a permis, d'une part, de mettre en place une méthode de documentation des aspects immatériels des objets et, d'autre part, d'ajouter des champs d'information au système documentaire Info-Muse. Pour accroître la diffusion des données de l'inventaire, la Chaire a conclu une entente avec le groupe Enseigner l'ÉCR! (acronyme pour le nouveau cours introduit dans les écoles du Québec intitulé «Éthique et culture religieuses ») de la Faculté de théologie et de sciences religieuses de l'Université Laval, regroupement de différents intervenants en éthique et culture religieuses (chargés de cours, professeurs, chercheurs, collaborateurs) pour l'utilisation des données du site de l'IPIR à des fins pédagogiques. Le mandat du groupe de recherche est d'offrir des ressources en réponse à des besoins exprimés par des enseignants et complémentaires à ce qui est par ailleurs disponible.

La deuxième mesure a été le rapprochement avec les communautés détentrices du patrimoine immatériel en multipliant autant que possible les contacts avec elles. Une lettre d'invitation à participer au projet de l'inventaire était envoyée aux communautés choisies. Par la suite, la coordonnatrice, parfois accompagnée des étudiants responsables des entrevues, organisait une rencontre avec les responsables de la communauté afin de présenter en détail le projet. Lors de la rencontre, le responsable de la communauté désignait une personne-ressource qui servait de guide dans la communauté. Ces premiers contacts ont permis d'associer les membres des communautés au projet et d'établir un climat de confiance. Lors des enquêtes orales, les étudiants mangeaient et logeaient avec les sœurs ou les prêtres, parfois pendant plusieurs jours, ceci dans le but de faire de l'observation participante et d'accroître la participation des membres des communautés. Ces collaborations très étroites ont permis souvent d'identifier sur place de nouveaux éléments à inventorier et de trouver de nouveaux usages pour ceux inventoriés.

Les interventions culturelles représentent le troisième moyen de participation. Ces interventions effectuées sur le terrain ont permis d'accroître la 
diffusion et la mise en valeur éducative et culturelle des ressources ethnologiques identifiées. Cette approche a eu des retombées immédiates au cours de la recherche de terrain dans la communauté et en dehors. Il ne s'agissait pas de faire œuvre d'évangélisation, ni de pratiquer de la pastorale, mais plutôt de présenter les pratiques cultuelles dans une perspective culturelle et d'assurer un retour de ces pratiques devenues patrimoine dans la communauté et la société élargie.

Les actions culturelles ont été organisées en trois temps. D'une part, directement sur le terrain, lors de la cueillette des données, par des présentations orales du travail effectué, par la remise de matériel audiovisuel aux communautés religieuses pour leurs sites Web particuliers ou pour la préparation d'expositions, par la publication d'articles dans les journaux, par la participation à des émissions de radio et de télévision, et par des conférences publiques. Les enquêteurs ont consacré entre le quart et le tiers de leur temps de terrain à des projets de mise en valeur élaborés en collaboration avec les membres des groupes religieux. Les activités culturelles pouvaient prendre plusieurs formes et se dérouler à différents moments du terrain selon les besoins et les attentes de la communauté. Les données recueillies et enregistrées sur disques (CDRom argent et or pour assurer une meilleure conservation) ont tout d'abord été déposées systématiquement aux archives des communautés pour utilisation ultérieure.

Pendant le terrain, les enquêteurs étaient parfois sollicités à participer à des activités déjà en cours de réalisation dans la communauté. Par exemple, dans la paroisse orthodoxe de Saint-Pierre et Saint-Paul, l'ethnologue a contribué au montage d'une exposition sur le centième anniversaire de la fondation de la paroisse. À la demande du conseil d'administration de l'église baptiste de Marieville, l'enquêteur a numérisé les photographies remises par les paroissiens lors d'une cueillette spéciale dans la paroisse. Des entrevues furent accordées aux journalistes pour des revues (par exemple la revue Notre-Dame-du-Cap), des journaux (Le Devoir, le journal autochtone Innuvelle), des bulletins, ou des émissions radiophoniques (par exemple, à la radio CKAU de Mani-Utenam, Radio Galilée, Radio-Canada, 3600 secondes d'histoire, CKIA, etc.). Les témoignages recueillis auprès des Ursulines (enregistrements audio et vidéo) ont été utilisés dans le cadre d'une exposition sur la mission éducative des Ursulines. Toujours pour les Ursulines, la reproduction en $3 \mathrm{D}$ du couloir de pierres du monastère a été présentée à la séance de clôture de l'Assemblée générale d'Icomos qui s'est tenue à Québec en $2008^{17}$. Une exposition multimédia couvrant l'ensemble des communautés

17. Ce document multimédia en 3 dimensions sur Dvd évoque l'esprit d'un lieu emblématique du patrimoine religieux de Québec, dans ses dimensions matérielles et immatérielles : le corridor de pierre du monastère des Ursulines. Cette réalisation a été présentée dans le cadre des activités de la 
religieuses du Québec a été créée et présentée à l'automne 2010 à l'Église Notre-Dame-de-Jacques-Cartier dans le quartier Saint-Roch de la ville de Québec ${ }^{18}$. La présentation du projet IpIR à l'émission Second Regard est sans doute l'action culturelle qui a eu le plus d'impact auprès du grand public. Second Regard, magazine à l'antenne de Radio-Canada, qui s'intéresse à la spiritualité et aux pratiques religieuses de toutes les cultures, est une émission très suivie par les téléspectateurs québécois. À l'hiver et au printemps 2010, le journaliste-réalisateur Jean-Robert Faucher et son équipe ont suivi sur le terrain des équipes de l'inventaire du patrimoine immatériel religieux pour préparer un reportage de 20 minutes qui a été diffusé à l'automne 2010 à Radio-Canada, en reprise sur RDi le même jour et le lendemain. Au cours des semaines qui ont suivi l'émission, les responsables de l'IPIR ont reçu de nombreux courriels de téléspectateurs. Selon Chiara Bortolotto, spécialiste européenne du patrimoine immatériel qui a étudié de près nos méthodes, une chaîne s'opère dans l'IPIR par la restitution des données aux communautés qui à leur tour les mettent en valeur : « Le principe de "retour", tel que défini par le projet, implique un transfert de ressources qui va du chercheur qui observe, détenteur du savoir scientifique, à l'acteur observé, détenteur de l'expression culturelle. Les connaissances des acteurs observés sont recueillies et traitées par les chercheurs, puis "retournées" à leurs "propriétaires" "d'une manière organisée" 19 ".

L'accessibilité au moyen d'un site Web (www.ipir.ulaval.ca) représente le deuxième temps de la valorisation. Les 880 fiches multimédia de la banque de données exposent les éléments de ce patrimoine sur plusieurs supports - textuels, iconographiques, audio et audiovisuels - permettant à chaque communauté d'identifier ses traditions et de mieux exploiter son potentiel culturel. En plus d'aider directement les communautés dans l'identification

$16^{\mathrm{e}}$ assemblée générale d'Icomos en septembre 2008 devant 750 congressistes provenant de 145 pays. Le programme d'interprétation multimédia et multisensoriel a été jugé très novateur et très bien reçu.

18. L'exposition « Bénis de Dieu » est sans doute celle qui a eu le plus d'impact. Préparée par l'ethnologue Marie Renier qui a travaillé à l'inventaire pendant quatre ans, avec l'aide des communautés, l'exposition a permis de mettre en valeur le matériel provenant de tout le Québec et d'englober divers aspects du patrimoine religieux, surtout dans son aspect immatériel. Les religieuses et religieux rencontrés lors de l'inventaire ont témoigné de l'expérience individuelle du sacré et expliqué aussi le sens et le devenir de ces pratiques. Elle s'est déroulée du 24 septembre au 21 novembre 2010 à l'église Notre-Dame-de-Jacques-Cartier à Québec (190, rue Saint-Joseph Est) et a attiré un grand nombre de visiteurs, dont les 400 congressistes du colloque annuel de la Société des musées québécois (SMQ), qui ont assisté à son inauguration le 13 octobre 2010 en présence de l'archevêque de Québec, M $^{\text {gr }}$ GéraldCyprien Lacroix.

19. Chiara Bortolotto et Isabelle Becuywe, «Inventari del patrimonio culturale immateriale in Québec ", dans AsPaCI (dir.), Identificazione partecipativa del patrimonio culturale immateriale transfrontaliero. Supporto scientifico al WP « Strategie per la salvaguardia e la valorizzazione dei patrimoni immateriali in applicazione della Convenzione UnESCO ». Progetto « E.CH.I. - etnografie italo svizzere per la valorizzazione del patrimonio immateriale ». P.O. di Cooperazione Transfrontaliera Italia Svizzera 2007-2013, 2010, p. 150 
de leur riche patrimoine immatériel, la mise en ligne des récits des communautés contribue à une meilleure connaissance des traditions religieuses qui ont façonné le Québec. Internet a permis de multiplier les différentes formes de mise en valeur de cette riche base de données multimédia : par la création et l'alimentation de sites thématiques pour les communautés, par le montage de documents multimédia sur des questions précises, par la préparation de matériel promotionnel pour le tourisme et par l'élaboration de programmes de formation. Par exemple, le site IPIR est ainsi devenu un outil pédagogique dans le cadre du programme d'Éthique et culture religieuses du ministère de l'Éducation, du loisir et du sport. Ce nouveau programme, instauré dans les écoles primaires et secondaires depuis 2008, adopte une approche culturelle du phénomène religieux : il permet de poser un regard sur le patrimoine religieux du Québec dans toutes ses dimensions (immobilier, mobilier et immatériel). Le site IPIR présente les traditions religieuses qui ont marqué le Québec (catholicisme, protestantisme, judaïsme et spiritualité autochtone), mais également des traditions religieuses anciennes (bouddhisme, islam, hindouisme, etc.) implantées plus récemment sur le territoire.

La diffusion scientifique constitue le troisième moyen de valorisation. L'inventaire représente un important corpus de données inédites dans lequel les étudiants et les professeurs ont puisé et continueront à puiser des informations pour préparer des conférences, des séminaires, des mémoires de maîtrise, des thèses de doctorat, des articles scientifiques et des livres savants et grand public $^{20}$. La diffusion scientifique joue un rôle très important, elle permet de faire connaître le patrimoine immatériel du Québec dans le milieu des spécialistes, tant sur le plan national qu'international, de le faire reconnaître par les communautés, d'accroître par l'analyse sa compréhension et sa connaissance pour tous, éléments indispensables à sa sauvegarde, tel que l'indique l'article 2.3 de la Convention pour la sauvegarde du patrimoine culturel immatériel21.

20. Parmi les travaux scientifiques les plus importants, citons : Laurier Turgeon et Louise SaintPierre, "Le Patrimoine immatériel religieux au Québec : sauvegarder l'immatériel par le virtuel », Ethnologies, 2009, vol. 31, no 1, p. 201-233 ; Laurier Turgeon et al., " Le Patrimoine ethnologique et les nouvelles technologies ", Khaldoun Zreik (dir.), Patrimoine 3.0, Actes du douzième colloque international sur le document électronique (CIDÉ 12), Paris, Europia, 2009, p. 1-14 ; Louise Saint-Pierre et Françoise Simard, « Dialogue sur le parvis : pour une approche intégrée du patrimoine religieux », Musées, vol. 29, 2010, p. 62 ; Daniela Moisa, « De l'intimité culturelle au patrimoine. La communauté russe orthodoxe de Rawdon ", Ethnologie française, 2010, vol. 59, n 3, p. 447-457; Mathilde Lamothe, "Captation d'images et inventaire multimédia du patrimoine immatériel : une expérience québécoise ", Journal des anthropologues, 2012, n ${ }^{\circ}$ 130-131, p. 305-329 ; Patricia Heiniger-Casteret, « Patrimoine culturel immatériel, deux expériences internationales : légiférer et transmettre », dans Rencontres internationales du patrimoine culturel immatériel en Bretagne, Actes des Rencontres de Brest les 14 et 15 décembre 2012, Rennes, Bretagne Culture Diversité et Dastum, 2014, p. 47-50 ; Valérie VachonBellavance, «La Patrimonialisation chez les Sœurs du Bon-Pasteur de Québec », mémoire de maitrise en ethnologie et patrimoine, Département des sciences historiques, Université Laval, 2014.

21. Les huit mesures de sauvegarde désignées sont : " l'identification, la documentation, la recherche, la préservation, la protection, la promotion, la mise en valeur et la transmission », Convention 
L'inventaire s'inscrit dans les mesures de documentation et de recherche, qui sont parmi « les premières stratégies que les États envisageront en vue de comprendre « ce qu'il y a », « qui le fait» et «pourquoi ils le font ${ }^{22}$ ».

Dans la perspective de poursuite de l'inventaire, il serait possible d'accroître encore la participation et de rendre les communautés plus autonomes en matière d'inventorisation du patrimoine immatériel en mettant à contribution le personnel des centres d'archives et des musées qui se trouvent à l'intérieur des communautés religieuses. En dépit de quelques tentatives pour faire participer les sœurs ou les prêtres directement aux enquêtes, soit dans la prise de notes, dans la captation d'images et dans le montage de vidéos, aucun n'a voulu s'engager en tant qu'enquêteur : ces religieux s'estimaient trop âgés, ne disposaient pas de beaucoup de temps et ne manifestaient pas un grand intérêt pour ce type de travail somme toute très spécialisé et technique. En revanche, il serait très possible de former le personnel, plus jeune et motivé, des dépôts d'archives et des musées religieux. Un grand nombre de monastères possèdent des centres d'archives et des musées qui emploient très souvent de jeunes ethnologues, archivistes et muséologues. Si ceux-ci possédaient une bonne formation de base en ethnologie et patrimoine, ils pourraient produire des données ethnographiques et audiovisuelles très utiles pour la réalisation d'un inventaire multimédia du patrimoine immatériel. Il faudrait former ces personnes au patrimoine culturel immatériel, à sa sauvegarde et aux pratiques d'inventorisation (identification, entrevues, captation audio et vidéo, montages, mise en ligne ou conservation selon le cas). Ces formations pourraient être organisées en collaboration avec Mission patrimoine religieux (MPR). Étant sur place, ces jeunes laïcs seraient bien placés pour former éventuellement des religieux intéressés à acquérir une spécialisation dans l'inventorisation du patrimoine immatériel.

Cependant, il faut être conscient que, pour produire de bons résultats, ces formations devront se faire sur plusieurs semaines, voire sur plusieurs mois, exiger des expériences de terrain et mobiliser des ressources financières importantes. Et il faudrait recommencer la formation dans chaque communauté. Cette approche serait davantage conforme aux orientations de l'UNESCO qui encourage l'inventorisation directement par les communautés, mais celles-ci font actuellement elles-mêmes l'objet de débats et de remises en cause. Bien que la Section du patrimoine culturel immatériel de l'UNESCO ne réalise pas d'inventaires, une responsabilité dévolue aux États parties, elle a créé un programme de renforcement des capacités qui comprend des ateliers d'inventorisation. Appelés « Inventaire basé sur les communautés », ces ateliers d'une semaine proposent, à l'aide de deux formateurs experts de

pour la sauvegarde du patrimoine culturel immatériel, Paris, UNESCO, 2003.

22. Identifier et inventorier le patrimoine culturel immatériel, Paris, UnEsco, 2010, p. 5. 
la Convention, une formation de base sur le patrimoine immatériel, sur le contenu de la Convention et sur l'approche à l'inventorisation prônée par l'UNESCO, soit celle de la participation des communautés de praticiens. En plus de fournir des informations de première main sur la pratique, les praticiens sont appelés à identifier les éléments à inventorier et également à participer à la cueillette et à l'analyse de l'information. Cette approche dite " par le bas » vise à inverser les rôles de manière à ce que l'expert ou le chercheur accompagne l'informateur et que l'informateur devienne à la fois auteur et acteur de l'inventorisation de sa pratique ${ }^{23}$. L'approche n'est pas sans susciter des discussions et des débats. Si les militants d'ONG et d'organisations associatives accueillent généralement assez bien ce tournant, le considérant comme une victoire de la démocratie participative, les scientifiques et les professionnels du patrimoine la voient généralement comme un discours démagogique, romantique et dangereusement «populiste », pour reprendre une expression de Chiara Bortolotto ${ }^{24}$. Le débat est loin d'être terminé et les expériences concrètes de terrain contribueront certainement à évaluer le bien-fondé des différentes approches et de développer un protocole de bonnes pratiques en matière d'inventorisation.

L'expérience de l'IPIR a démontré qu'il y a des limites à l'approche participative de l'inventorisation et qu'il sera utile de les faire connaître. Même si cette approche peut contribuer à augmenter l'engagement des praticiens dans la sauvegarde de leur patrimoine immatériel, elle peut aussi nuire à la qualité de l'inventaire, restreindre son utilisation et faire augmenter considérablement les coûts de sa production. Il faut rappeler que l'inventorisation est un métier en soi qui exige des savoir-faire particuliers et de l'expertise que les praticiens de l'inventaire développent progressivement au cours de leur pratique. C'est sans doute pour cette raison que dans la plupart des autres domaines, que ce soit en histoire, en architecture ou en filmographie, les inventaires sont réalisés par des spécialistes ayant reçu une formation en archivistique ou en bibliothéconomie, souvent avec plusieurs champs de compétences. Pour faire

23. Il est possible de s'inscrire sur le site de l'UnESCO pour avoir accès aux trousses de formation : www.unesco.org/culture/ich/index.php?lg=fr\&pg=00679. J'ai eu l'occasion d'assister à un atelier de formation en inventorisation organisée par l'UNESCO au Cap Haïtien en Haïti du 13 au 17 mai 2013. Il m'est difficile d'évaluer le bien-fondé et l'efficacité de ce programme à partir de cette expérience isolée, d'autant que dans ce cas la grande majorité des trente participants inscrits étaient des étudiants universitaires, des fonctionnaires et des élus ; seulement deux ou trois étaient des praticiens. Il est sûr qu'un atelier de cinq jours n'a pu être plus qu'une sensibilisation au patrimoine immatériel, à l'inventaire et aux mesures de sauvegarde, plutôt qu'un réel apprentissage des méthodologies et des techniques de l'inventaire avec une sortie sur le terrain. D'après l'expérience de l'IPIR, un inventaire multimédia possédant un bon contenu scientifique exige plusieurs mois, voire plusieurs années, de formation et de pratique.

24. Chiara Bortolotto, "The French Inventory of Intangible Cultural Heritage : Domesticating a Global Paradigm into French Heritage Regime », dans Regina F. Bendix, Aditya Eggert, Arnika Peselmann (dirs.), Heritage Regimes and the State, Gottingen, Universitätsverlag Göttingen, series « Göttingen Studies in Cultural Property », vol. 6, 2012, p. 269. 
un inventaire multimédia du patrimoine immatériel religieux, possédant un bon contenu scientifique et visuel, il est essentiel d'avoir des compétences poussées dans au moins quatre domaines : 1) l'histoire et l'ethnologie des religions, 2) l'enquête ethnologique, 3) la photographie et la vidéographie, et 4) la communication multimédia. Non seulement faut-il plusieurs années de formation et d'expérience de terrain pour acquérir ces compétences, mais il est rare qu'une même personne puisse toutes les réunir à elle seule. Selon l'expérience de l'IPIR, la constitution d'une équipe interdisciplinaire, composée de deux personnes, un ethnologue et un vidéaste/photographe, produisait les meilleurs résultats à des coûts raisonnables. L'ethnologue se chargeait de préparer les entrevues, de les réaliser, de rédiger les guides d'écoute et les fiches de l'inventaire alors que le vidéaste/photographe s'occupait de prendre les photos, de capter l'entrevue à l'aide de la caméra vidéo et de faire le montage de la capsule vidéo. En juin 2011, nous avons organisé une université d'été à Saint-Jean-Port-Joli avec l'intention de former des praticiens à l'inventorisation. En dépit d'une excellente publicité faite dans la région et de notre bonne volonté, il n'a pas été possible de recruter des praticiens pour la formation. Certains se sont inscrits pour la formation générale, mais aucun n'a voulu apprendre à conduire des entrevues ou à manier la caméra vidéo, trouvant ces tâches techniques trop compliquées; ils préféraient consacrer ce temps à la pratique de leur propre métier. C'est alors que l'on a réalisé qu'il faudrait des heures d'enseignement et de pratique et des budgets très importants pour former un praticien à inventorier convenablement sa seule pratique et peut-être celle de certains de ses collègues. Les coûts d'une telle aventure seraient prohibitifs. De plus, le regard distancé de l'ethnologue contribue à donner plus de recul et de profondeur au recensement de la pratique, puis une perspective comparative, qui est absente lorsqu'elle est inventoriée par un praticien parfois trop collé sur sa manière particulière de faire et tenté de survaloriser sa pratique par rapport à celle des autres, le mettant automatiquement en conflit d'intérêt avec d'autres praticiens.

Les organismes ou individus qui ont pratiqué l'auto-inventorisation, soit la réalisation d'inventaires par les praticiens eux-mêmes, se sont vite rendu compte des limites d'une telle approche. Après avoir commencé à élaborer un inventaire très sommaire sous forme d'une simple liste de pratiques par des représentants de communautés ${ }^{25}$, la Belgique flamande a rapidement réalisé le caractère peu utile de l'exercice et a développé un inventaire multimédia en ligne semblable à celui de l'IPIR dont la gestion a été confiée à une ONG

25. Dries Van Den Broucke, «The Flemish Community : Supporting intangible cultural heritage through a network of intermediate organizations », dans Antoine Gauthier (dir.), Les Mesures de soutien au patrimoine immatériel, Québec, Conseil québécois du patrimoine vivant, 2012, p. 113-120, notamment p. 115. 
spécialisée dans la sauvegarde du patrimoine, Tapis plein ${ }^{26}$. De même, la France qui a tenté de telles approches à titre expérimental s'est tournée, par des appels d'offres, vers un ensemble varié de centres de recherches universitaires, d'associations professionnelles et d'instituts de métiers d'art, tout en s'inspirant des méthodes développées par la Chaire de recherche du Canada en patrimoine ethnologique au Québec, soit dans le cadre des inventaires IPIR et IREPI ${ }^{27}$. La région Aquitaine en France a lancé son propre inventaire régional dans un esprit de collaboration entre un laboratoire universitaire, l'ITEM de l'Université de Pau et des Pays de l'Adour, et un opérateur régional d'action culturelle occitane, InOc Aquitaine, tout en associant à titre de partenaire la Chaire de recherche du Canada en patrimoine ethnologique de l'Université Laval afin de développer ses approches. En effet, Patricia Heiniger-Casteret, professeur d'ethnologie à l'Université de Pau et directrice de l'inventaire d'Aquitaine, précise que « les premiers travaux d'inventaire en Aquitaine ont bénéficié de la réflexion pionnière portée par l'Université Laval qui, depuis 2004, a élaboré des outils méthodologiques ${ }^{28}$ ». Patricia Heiniger-Casteret travaille avec les différents organismes et praticiens du patrimoine immatériel en Aquitaine depuis de nombreuses années. Après avoir fait des efforts pour leur faire pratiquer l'inventaire, elle est parvenue à des conclusions proches des nôtres, soit que les praticiens n'étaient pas réellement intéressés et qu'il s'agissait d'une orientation inutilement compliquée et coûteuse ${ }^{29}$. On pourrait ajouter à cette liste d'exemples celui de la Suisse qui avait préconisé la constitution d'un inventaire du patrimoine immatériel avec la participation d'organismes culturels cantonaux, mais qui, en raison de contraintes de temps et d'argent et d'un évident besoin de cohérence, a finalement eu recours à des spécialistes ${ }^{30}$.

26. Ellen Janssens, Hans van der Linden et Bram Wiercx, "www.immaterieelerfgoed.be : A plateforme for intangible cultural heritage in Flanders (Belgium) ", dans Digital Heritage Conference Proceedings, Marseille, CNRs, 2013, tome 1, p. 96.

27. Voir, Sylvie Grenet, «Les Inventaires en France », Culture et Recherche, 2008, $\mathrm{n}^{\text {os }} 116-117$, p. 27-28 ; Sylvie Grenet, "Les Inventaires en France », dans Christian Hottin (dir.), Le Patrimoine culturel immatériel : premières expériences en France, Internationale de l'imaginaire-nouvelle série, 25, Paris, Babel, 2011, p. 77-92; et Chiara Bortolotto, «The French Inventory...», op. cit., 2013, p. 271-273.

28. Patricia Heiniger-Casteret, op. cit., p. 47-48.

29. Entrevue avec Patricia Heiniger-Casteret, directrice du projet d'inventaire du patrimoine immatériel de l'Aquitaine et professeur d'ethnologie à l'Université de Pau et des Pays de l'Adour, le 17 mai 2014 à Pau, France.

30. Florence Graezer-Bideau, "Identifying "Living Traditions" in Switzerland : Re-enacting Federalism through the UnESCO Convention for the Safeguarding of Intangible Cultural Heritage ", dans Regina F. Bendix, Aditya Eggert, Arnika Peselmann (dirs.), Heritage Regimes and the State, Göttingen, Universitätsverlag Göttingen, series « Göttingen Studies in Cultural Property », vol. 6, 2012, p. 306-307 ; et informations extraites de l'entrevue avec Florence Graezer-Bideau, directrice du Centre for Area and Cultural Studies (CACS) - EPFL, et membre du comité scientifique de l'inventaire du patrimoine immatériel de la Suisse, le 22 mai 2014 à Neuchâtel, Suisse. 
Il existe de multiples manières de faire participer la communauté à la sauvegarde du Pci, par la transmission, par la sensibilisation, par l'usage, par l'adaptation des pratiques à des nouveaux usages sociaux et par la mise à sa disposition d'un inventaire qui lui sera accessible. Selon notre expérience, ces modes de participation s'avèrent plus productifs qu'une participation directe à la fabrique de l'inventaire qui est un métier en soi.

\section{L'approche par récits : forces et limites}

Nous avons opté pour une approche qui privilégie la cueillette des données par le récit oral et le film ethnographique, c'est-à-dire par la voix et l'image. Développée spécialement pour ce projet d'inventaire, l'enquête par le récit oral représente une forme d'entrevue semi-dirigée qui offre l'avantage de laisser l'informateur parler librement et facilement de ce qu'il sait et de ce qu'il a vécu. Nous inspirant de l'approche bien connue et répandue des récits de $v^{31}{ }^{31}$, nous avons voulu l'étendre aux objets, aux lieux et aux pratiques. Il s'agissait tout simplement de demander à l'informateur de parler d'objets, de lieux et de pratiques : leur histoire, leur signification, leurs modes de fabrication, leurs usages et leur valeur patrimoniale. En plus d'évoquer des expériences concrètes, les récits ont permis de faire le lien entre le patrimoine immatériel et matériel. En effet, il était plus facile pour les informateurs de parler du patrimoine à partir d'un objet concret, d'un lieu précis, d'une pratique significative ou de leur vie. Cette approche permettait de plonger les informateurs directement dans leurs usages du patrimoine immatériel et dans les modalités de sa fabrication plutôt que de leur faire à chaque fois un long cours d'introduction sur le patrimoine immatériel. Les objets et les lieux servent de soutien et de stimuli à la mémoire, d'outil mnémonique facilitant le rappel des modes de fabrication, des usages et du sens donné aux biens patrimoniaux. Le récit facilite le va-et-vient entre le sens et la chose, entre la pratique et l'objet, entre l'immatériel et le matériel, et la conception même du patrimoine immatériel.

Cette approche s'appuyait sur les catégories apparaissant dans la définition du patrimoine culturel de la Convention pour la sauvegarde du patrimoine culturel immatériel de l'Unesco et permet de faire le lien entre les pratiques, les personnes, les lieux et les objets. En effet, la Convention précise : « On entend par "patrimoine culturel immatériel” les pratiques, représentations, expressions, connaissances et savoir-faire - ainsi que les instruments, objets, artefacts et espaces culturels qui leur sont associés - que les communautés,

31. Voir notamment Daniel Bertaux, Récits de vie, Paris, Nathan, 1996 ; Patrick Brun, Émancipation et connaissance. Les histoires de vie en collectivité, Paris, L'Harmattan, 2001 ; le Réseau québécois pour la pratique des histoires de vie (www.rqphv.org) ; et Carole Dornier et Renaud Dulong (dir.), Esthétique du témoignage, Paris, Éditions de la Maison des sciences de l'homme, 2005. 
les groupes et, le cas échéant, les individus reconnaissent comme faisant partie de leur patrimoine culturel ». Il faut souligner aussi que nous nous sommes inspirés de la catégorisation du Programme de proclamation des chefs-d'œuvre du patrimoine oral et immatériel de l'humanité (2001-2005) qui était en cours à l'époque de la conception de l'IPIR de 2006 à 2007. Ce programme classait les chefs-d'œuvre selon trois catégories : porteur de tradition, espace culturel et forme d'expression ${ }^{32}$. Nous avions alors la volonté de nous conformer au système de l'UNESCO qui représente la référence internationale et qui établit les normes en matière de patrimoine immatériel.

Ainsi, nous avons défini les quatre catégories de récits de la façon suivante :

1. les récits de lieux portent sur la construction, l'usage et le sens des espaces les plus significatifs dans chacune des communautés, les hauts lieux de l'habitat (chapelle, sacristie, jardin, grotte, réfectoire, salle d'enseignement, cimetière, presbytère, synagogue, lieu de culte, espace communautaire) ;

2. les récits d'objets renvoient aux modes de fabrication, aux usages et aux sens des objets matériels ayant une forte valeur symbolique et identitaire, et jugés les plus significatifs pour les communautés sur le plan patrimonial (objet religieux, vêtement liturgique, habit traditionnel, mobilier traditionnel, mobilier de cuisine, etc.) ;

3. les récits de vie visent à documenter la vie ou des épisodes de vie de membres de la communauté renfermant un caractère exemplaire et donc une valeur patrimoniale (missionnaire, artiste, artisan, enseignant, etc.) ;

4. les récits de pratiques cultuelles et culturelles regroupent les dévotions particulières, les coutumes funéraires, les pratiques liturgiques significatives, les pratiques professionnelles marquantes, les savoir-faire artistiques et artisanaux uniques ayant une valeur à la fois pragmatique et symbolique dans la communauté (la statuaire, la broderie, la dentellerie, la dorure, le tressage, la fabrication d'objets religieux, la fabrication de produits alimentaires, etc.).

Les catégories choisies pour inventorier le patrimoine immatériel religieux se sont avérées pertinentes. Toutes les données recueillies lors des entrevues ont été traitées sans difficulté dans l'une ou l'autre des catégories. Dans le cas d'un chevauchement de plusieurs types de récits dans la même entrevue, les enquêteurs ont rédigé des fiches d'inventaire pour chaque type de récit. Cette approche a permis de consulter une pratique, par exemple les rites funéraires d'une communauté sous différents aspects : la symbolique du cercueil (récit d'objet), la préparation du corps du défunt (récit de pratique), les funérailles (récit de pratique) et le cimetière (récit de lieu). Lorsque différents infor-

32. Voir à ce sujet le site l'UNESCO : www.unesco.org/culture/ich/index.php?pg=00103\&lg=FR. Cette information a été confirmée par Rieks Smeets, ancien chef de la section du patrimoine culturel immatériel à l'UNESCO (2000-2008), lors d'une entrevue le 28 mars 2012 à Bruxelles. 
mateurs ont abordé le même sujet, une fiche individuelle a été complétée. Les fiches d'inventaire sont descriptives et factuelles, incluent des données nominatives (nom, adresse de l'informateur, rôle dans la communauté, etc.), et des données techniques d'inventaire (nom de l'enquêteur, indexeur, documents audio et vidéo, date des entrevues et du traitement, etc.). Chaque récit fait l'objet de descriptions textuelles : l'historique et la description de la pratique, son actualisation ainsi que ses modes de transmission. Dès le départ, nous avons privilégié une approche méthodologique permettant d'aller au-delà de la simple description; nous voulions rendre compte du vécu du patrimoine au sein de la communauté. Nous avons filmé autant que possible les entrevues, surtout sur les objets, les lieux et les pratiques, afin de lier la parole à l'image, la pratique à l'objet ou au lieu, et d'offrir l'illustration la plus concrète et vivante.

Quoique très originale et efficace, l'inventorisation du patrimoine immatériel religieux par récit ne devrait pas être poursuivie pour la bonne raison que l'Unesco n'utilise plus ces catégories. En effet, l'UnEsco a abandonné son programme des proclamations et l'a remplacé en novembre 2008 par celui des listes, la Liste représentative et la Liste de sauvegarde urgente. Par la même occasion, il a abandonné les trois catégories employées pour le classement des proclamations (porteur de tradition, espace culturel et forme d'expression qui avait inspiré notre catégorisation initiale) pour retenir une seule catégorie pour le classement, soit celle de forme d'expression, ou celle mieux connue maintenant sous le nom de «pratique ». Voulant privilégier les communautés et les formes pérennes, l'UNESCO a jugé opportun de ne classer sur les nouvelles listes que des pratiques, davantage collectives et transmises de génération en génération, offrant donc de plus grandes chances de perdurer. La catégorie « porteur de tradition » renvoie à des individus plutôt qu'à des communautés, et leurs pratiques peuvent disparaître avec eux. Les espaces culturels posaient aussi problème pour l'UNESCO car, plus difficiles à délimiter, ils dédoublaient le programme des sites du patrimoine mondial régi par la Convention du patrimoine mondial, culturel et naturel, de $1972^{33}$. Dans la mesure où l'UNESCO n'accepte plus que les pratiques collectives pour le classement sur les listes et qu'il établit les normes au niveau international, il vaudrait mieux ne retenir pour la poursuite de l'inventaire qu'une seule catégorie, soit celle des pratiques. Cette transformation ne devrait pas poser problème car, de toutes les façons, la plupart des récits recensés dans l'IPIR renvoient à des pratiques. D'abord, la pratique était déjà présente dans l'IPIR

33. Entrevue avec Rieks Smeets, ancien chef de la section du patrimoine culturel immatériel à l'UnESCo (2000-2008), le 28 mars 2012 à Bruxelles; deux entrevues avec Cécile Duvelle, nouvelle chef de la section du patrimoine culturel immatériel de l'UNESCo depuis 2008, le 27 mars 2012 et le 14 mai 2014 à Paris. 
sous le nom de « forme d'expression » qui regroupe les deux tiers des récits, soit $66 \%$. Ensuite, les récits de lieux et les récits d'objets, qui représentent respectivement $14 \%$ et $11 \%$ du total des récits, décrivent souvent des savoirfaire liés à la fabrication ou à l'usage des objets et des lieux, donc des pratiques. Même les récits de vie (9\% des récits) évoquent parfois des pratiques du porteur de tradition. Il sera donc possible de reprendre et de retravailler les textes pour inscrire ces récits comme des pratiques, si le MCCQ souhaitait se conformer à la lettre aux modes de fonctionnement de l'UNESCO. C'est d'ailleurs ce que l'UNESCO a fait lorsque les 90 proclamations des chefs-d'œuvre ont été intégrées à la liste représentative en 2008.

Toutefois, il est important de rappeler que la Convention pour la sauvegarde du patrimoine culturel immatériel de l'UNESCO ne donne pas de directives strictes sur les manières de faire des inventaires, laissant aux États parties le loisir de choisir le mode d'inventorisation qui leur convient le mieux. Si la Convention exige que les États parties réalisent des inventaires avant de proposer des éléments pour le classement sur les listes, l'article 12.1, consacré aux inventaires, précise que, " pour assurer l'identification en vue de la sauvegarde, chaque État partie dresse, de façon adaptée à sa situation, un ou plusieurs inventaires du patrimoine culturel immatériel présent sur son territoire ». L'expression, « de façon adaptée à sa situation », introduit un élément de souplesse et invite à la créativité et à la diversité. Il faut préciser que la Section du patrimoine culturel immatériel de l'UNESCO ne dresse pas d'inventaires ; elle administre le respect des principes de la Convention, organise l'évaluation de candidatures et gère les listes. Son expérience en matière d'inventorisation est restreinte aux observations faites sur les inventaires des pays signataires et non à une réelle pratique de l'inventaire.

Il convient aussi de rappeler qu'un inventaire et une liste sont deux choses très différentes, chacun avec sa propre vocation et sa spécificité. Un inventaire est essentiellement un outil de connaissance et de sauvegarde. Comme l'indique l'article 12.1 de la Convention, il vise à « assurer l'identification en vue de la sauvegarde ». Par conséquent, il doit recenser dans le moindre détail le plus grand nombre possible de pratiques locales, d'une part, pour identifier celles qui sont menacées dans l'optique de leur sauvegarde, et, d'autre part, pour tenir compte des variations des pratiques entre les groupes et les localités. Très souvent, les groupes et les régions qui partagent une même pratique introduisent des manières de faire différentes, parfois subtiles, néanmoins importantes, car celles-ci leur permettent de se distinguer les uns des autres et d'affirmer leur identité. L'inventaire permet ainsi de tenir compte et d'entretenir la diversité des cultures sur un territoire, voire même d'encourager leur développement, ce qui rejoint l'objectif principal de la Convention - faut-il le rappeler. La liste, elle, possède une autre fonction, 
celle de réunir plusieurs groupes qui partagent une pratique pour la faire reconnaître. Sélective par définition, la liste incite les groupes partageant une même pratique à se regrouper pour poser une candidature de façon collective et accroître les chances de succès. D'ailleurs, l'UNESCO encourage les régions et les pays à agir en ce sens, soit à se réunir pour préparer des candidatures communes dans le but de contribuer au renforcement de la cohésion sociale, pour accroitre l'harmonie et le bonne entente entre les pays, et pour maintenir la paix dans le monde, valeurs qui sont celles de l'UnesCo. Sinon, le classement sur les listes aurait un effet contraire, soit de stimuler la concurrence, les conflits et éventuellement les affrontements.

\section{La grille de classification de Jean Du Berger : regards réflexifs}

Nous avons été rapidement confrontés à la mise en place d'un système de classification logique et cohérent qui régularise le lexique utilisé pour l'inventorisation des éléments du patrimoine culturel immatériel. Contrairement à la culture matérielle qui bénéficie de systèmes de classification, comme celui de Chenhall largement employé en Amérique du Nord ${ }^{34}$, il n'existe pas d'équivalent pour la culture immatérielle en raison du développement très récent de ce champ de connaissances. Pour y remédier, nous avons repris et adapté la grille des pratiques culturelles de Jean Du Berger ${ }^{35}$. Professeur d'ethnologie à l'Université Laval, Jean Du Berger l'avait développée comme un outil d'analyse du fonctionnement culturel. Il n'en demeure pas moins qu'elle s'est avérée être aussi un outil de classification, car cette grille relationnelle permet d'évoquer les rapports entre les différentes pratiques culturelles et de montrer leur organisation et leur fonctionnement en société. La grille est divisée en trois champs, le champ coutumier, le champ pragmatique et le champ symbolique et expressif. Ces champs forment un réseau, le champ coutumier incluant pour ainsi dire les autres champs puisque la coutume est le fondement de tous nos comportements et de notre vie en société.

Dans la grille des pratiques culturelles, les objets et les pratiques sont interreliés. Par exemple, les objets de dévotion pourront être considérés sous l'aspect de leur fabrication (champ pragmatique) ou de leur utilisation dans un rituel (champ symbolique et expressif). Ainsi les champs sont interactifs et les catégories non exclusives, ce qui représentait un avantage dans un inventaire informatisé sur le patrimoine immatériel religieux inclusif et interrelationnel. Certaines pratiques, par exemple celles des associations caritatives

34. Robert G. Chenhall, Nomenclature for Museum Cataloguing : A System for Classifiing Manmade Objects, Nashville, Tennessee, American Association for State and Local History, 1978 ; James A. Blackaby, Patricia Green et le Nomenclature Committee, The Revised Nomenclature for Museum Cataloguing : A Revised and Expanded Version of Robert G. Chenhall's System for Classifying Man-Made Objects, Nashville, Tennessee, American Association for State and Local History, 1988.

35. Jean Du Berger, op. cit. 
laïques (champ coutumier), ont souvent un lien étroit avec des communautés religieuses et avec le religieux (champ symbolique). S'il ne s'agit pas véritablement de pratiques éthiques au sens strict, elles sont néanmoins inspirées d'une représentation du monde et d'un système de croyances.

Les pratiques éthiques font partie du champ symbolique et expressif qui renferme également les pratiques ludiques et esthétiques, les pratiques langagières et les pratiques ethno-scientifiques. Selon Du Berger, les pratiques éthiques ont pour objet « la compréhension et l'explication des phénomènes qui dépassent le domaine naturel par des systèmes de croyance, l'encadrement des rapports de l'homme avec ces phénomènes dans une organisation religieuse et un culte, leur contrôle par l'homme grâce à des pratiques rituelles, la régulation des conduites humaines en fonction de ces phénomènes par un ensemble de prescriptions et d'interdits ${ }^{36} \gg$. Le champ des pratiques éthiques comprend neuf sous-champs : le système de croyances, l'organisation religieuse, la pratique religieuse, la pratique rituelle, la prescription et l'interdit, la magie, la sorcellerie, les pratiques religieuses alternatives et les superstitions. Chacun de ces sous-champs se décline en sous-éléments. Par exemple, le sous-champ pratique religieuse comprend cinq sous-éléments - culte, pratique langagière religieuse, enseignement religieux, pratique spirituelle et pratique ascétique - alors que le sous-champ pratique rituelle n'en comprend que trois - objet rituel, élément utilisé dans un rituel et geste rituel ${ }^{37}$.

L'expérience du terrain du projet pilote a servi de banc d'essai et fut l'occasion de tester l'opérabilité du système de classement. La réflexion sur le classement des récits s'est poursuivie tout au long du processus de traitement de l'information pendant et après le projet pilote. Les résultats de classification des pratiques religieuses nous ont donné l'occasion de connaître les limites et les contraintes de la grille de Du Berger. Si près de $95 \%$ des récits se classaient dans les quatre premiers niveaux de la grille, au-delà, il était difficile de classer les pratiques; des ajustements à la grille étaient également nécessaires afin de ne pas dénaturer les rites non chrétiens. Il nous est apparu pertinent de modifier l'appellation de certains niveaux pour permettre le classement des pratiques d'autres confessions (par exemple, « curé, rabbin, chef spirituel »). Il s'agissait en quelque sorte de « décatholiciser » la grille en utilisant des termes plus neutres et en y faisant au besoin certains ajouts.

De concert avec le MccQ, la Chaire a développé un outil de classification des éléments du patrimoine culturel immatériel en s'inspirant de la grille des pratiques culturelles de Du Berger : la classification a été réduite à quatre niveaux, certains concepts ethnocentriques ont été abandonnés (sorcellerie,

36 Ibid., p. 37.

37. Pour l'arborescence du champ des pratiques éthiques selon cette grille, voir Jean Du Berger, op. cit., p. 37. 
superstition, magie), privilégiant un lexique ethnologique reconnu afin de donner un caractère universel à la grille (les rites tels que définis : rite de passage, rite de sollicitation, etc.). Le champ des pratiques éthiques a été rendu plus exclusif et autonome, c'est-à-dire que les pratiques reliées à un système de croyance ou inspirées d'une représentation du monde ont été classées dans le champ des pratiques éthiques. Il est ainsi possible de classer les pratiques coutumières des groupes religieux, les pratiques alimentaires ou vestimentaires, les pratiques techniques reliées à des savoir-faire, l'organisation et les structures des groupes religieux dans un même champ ${ }^{38}$. Grâce à ces modifications, nous avons réussi à faire de la grille de classification du patrimoine culturel immatériel un outil opérationnel de classement.

Il n'en demeure pas moins que la grille a posé de nombreux problèmes. Elle a le défaut de sa vertu, soit son caractère très sophistiqué. Son utilisation exige une bonne maîtrise de la typologie qui demande une période assez longue d'apprentissage en raison de ses nombreux champs et niveaux. Le morcellement poussé des catégories et sous-catégories rend souvent difficile le classement des récits et des pratiques qui chevauchent plusieurs de cellesci. Ainsi, le choix du classement est parfois subjectif et arbitraire. Il a fallu consacrer beaucoup de temps à former les enquêteurs pour la manier. Il va sans dire que cette grille très rigide oriente la définition des différents champs des pratiques culturelles et la conception même du patrimoine immatériel pour lequel elle n'a pas été créée à l'origine. La grille est encore beaucoup plus difficile à utiliser comme outil de recherche dans la base de données pour l'internaute qui n'est pas forcément familier avec sa terminologie et encore moins avec sa logique et son arborescence. En somme, elle ne permet pas de classer et de retirer les pratiques à partir de catégories clairement définies et faciles à identifier par tout le monde. Il faut dire aussi que les moteurs de recherche informatiques sont devenus très efficaces, permettant aux communs des mortels de trouver instantanément de l'information par mot clé, par catégorie du patrimoine, par localisation géographique ou par confession religieuse. Il n'est donc plus indispensable de disposer d'un système de classification pour structurer et accéder à l'information comme lorsque l'on devait faire des recherches manuellement dans des fichiers de papier.

C'est sans doute pour ces raisons que la grille de Jean Du Berger n'a pas été reprise par d'autres projets d'inventaires dans le monde, qui ont pourtant tenté de l'adapter mais sans succès. Ce fut le cas pour la Belgique francophone (Wallonie/Bruxelles) comme pour la France qui a étudié la possibilité de l'utiliser dans son inventaire national. Le projet a été assez rapidement

38. Voir le site IPIR pour la grille de classification des éléments du patrimoine culturel immatériel développée à partir de la grille de Jean Du Berger (www.ipir.ulaval.ca). 
abandonné, car la grille a été jugée inutilement compliquée ${ }^{39}$. La grille n'a pas retenu l'attention des spécialistes de l'inventaire de la Suisse non plus pour les mêmes raisons ${ }^{40}$.

La plupart des inventaires du patrimoine culturel immatériel développés par les différents pays signataires de la Convention de l'UNESCO ont repris ou se sont inspirés des cinq domaines identifiés dans l'article 2.2 de la Convention, soit : 1) les traditions et expressions orales ; 2) les arts du spectacle ; 3) les pratiques sociales, rituels et événements festifs ; 4) les connaissances et pratiques concernant la nature et l'univers ; 5) les savoir-faire liés à l'artisanat traditionnel. La Suisse, pour son inventaire appelé Liste des traditions vivantes, a repris à peu près intégralement les domaines proposés par l'UNESCO : expressions orales, arts du spectacle, pratiques sociales (qui comprennent les fêtes et les rituels), nature et univers, et artisanat traditionnel ${ }^{41}$. Lorsqu'une pratique chevauche plusieurs catégories, on indique la catégorie dominante en premier et les autres par ordre décroissant d'importance. Présenté en cinq langues (français, allemand, italien, anglais et romanche, le site de l'inventaire de la Suisse offre la possibilité de faire des recherches par une liste de mots clés qui renvoient à des actions : danser et se mouvoir, façonner et décorer, voguer et flotter, jouer et lutter, conter et raconter, commémorer et célébrer, représenter et mettre en scène, etc. Quoique présentant son inventaire en une seule langue (le français), la France a été un peu plus audacieuse et novatrice car, en reprenant quatre des cinq catégories de l'UNESCO, elle en a scindé une en deux ; la catégorie des pratiques sociales, rituels et événements festifs de l'UNESCO a été divisée en deux (pratiques festives et pratiques rituelles, séparant ainsi les fêtes laïques des rituels religieux). Elle n'a pas retenu la catégorie des connaissances et pratiques concernant la nature et l'univers, du moins pas pour le moment. En revanche, la France a ajouté deux autres catégories : les jeux et les pratiques sportives ${ }^{42}$. La recherche sur le site de la France se fait par le biais de ces catégories qui rassemblent les éléments sous la forme d'une liste assez longue et laborieuse à consulter. L'Écosse a également ajouté quatre nouvelles catégories afin d'adapter le modèle UNESCO à ses propres réalités et volontés : traditions culinaires, danse et théâtre, musique et jeux ${ }^{43}$. La Belgique francophone (Wallonie/Bruxelles) sur

39. Entrevue avec Christian Hottin, Direction générale des patrimoines, Ministère de la Culture de la France, le 12 mai 2014 ; et entrevue avec Sylvie Grenet, chargée de mission pour le patrimoine culturel immatériel et le patrimoine ethnologique, responsable de l'inventaire du patrimoine immatériel, Ministère de la Culture de la France, le 17 mai 2014.

40. Entrevue déjà citée avec Florence Graezer-Bideau, le 22 mai 2014 à Neuchâtel, Suisse.

41. Voir le site de l'inventaire de la Suisse : www.traditions-vivantes.ch.

42. Voir le site de l'inventaire du patrimoine culturel immatériel de la France : www.culturecommunication.gouv.fr/Disciplines-et-secteurs/Patrimoine-culturel-immateriel/Inventaire-en-France/ Inventaire/Fiches-de-l-inventaire-du-patrimoine-culturel-immateriel.

43. Voir le site de l'inventaire de l'Écosse : www.ichscotlandwiki.org/index.php?title=Welcome. 
son site nommé « Patrimoine vivant Wallonie/Bruxelles » du « Programme Patrimoine Immatériel » réalisé en partenariat avec la Fédération Wallonie Bruxelles, a eu recours aux cinq catégories de l'UNESCO : artisans et métiers de luxe, connaissances et pratiques concernant la nature, arts du spectacle, pratiques sociales, rituels et fêtes, traditions et expressions orales ${ }^{44}$. Voulant mettre l'accent sur les pratiques de sauvegarde, la Belgique flamande a retenu, non pas les catégories contenues dans la définition du patrimoine immatériel de l'article 2.2 de la Convention de l'UnesCo, mais celles des mesures de sauvegarde qui apparaissent dans l'article 2.3 de la Convention, soit : l'identification et la documentation, la recherche, la communication et la promotion, la transmission et la revitalisation ${ }^{45}$. Ces catégories servent à la fois de classement des pratiques et d'outil de recherche. Plutôt que de créer des catégories en fonction de la nature de la pratique, le système de la Belgique flamande propose une classification par rapport aux mesures prises pour la sauvegarde de la pratique, mettant ainsi l'accent sur le travail de préservation et de revitalisation plutôt que sur la pratique elle-même. Cette approche novatrice est très récente, elle date de 2012 ; il sera intéressant de voir à l'essai sa fonctionnalité et son efficacité, et d'y mesurer les résultats dans quelques années.

Pour conclure, dans la perspective de la poursuite de l'inventaire du patrimoine immatériel religieux ou encore pour d'autres inventaires du patrimoine immatériel, il vaudrait mieux abandonner l'usage de la gille de Jean Du Berger, compte tenu des difficultés de son utilisation dans le projet IPIR, tant pour l'établissement du classement que pour la recherche dans la banque de données, et dans d'autres projets à l'international. Dans la mesure où la tendance chez les pays signataires de la Convention est de reprendre les catégories présentées dans la définition du patrimoine immatériel par l'UNESCO, il serait opportun pour le Québec de suivre ce mouvement qui devient une référence à l'international.

\section{Méthodologie de la diffusion des données multimédia : le site IPIR}

Le projet IPIR a privilégié, dès ses origines, une approche méthodologique permettant d'aller au-delà de la simple description ; nous avons ainsi rendu compte du vécu du patrimoine au sein des communautés. Nous avons exploité le plus grand nombre de médias possible, soit quatre (texte, photos, enregistrements audio et vidéo), pour transmettre avec le plus de précision et d'effi-

44. Plusieurs éléments du patrimoine culturel immatériel de la Belgique (31) sont répertoriés sur le site : www.patrimoinevivantwalloniebruxelles.be.

45. Je remercie Marc Jacobs, directeur de FARO (interface flamand du Centre pour le patrimoine culturel), entrevue du 18 mai et du 14 août 2014. Voir le site de la Belgique flamande : www.immaterieelerfgoed.be. 
cacité possible le caractère vivant de ce patrimoine, faciliter sa transmission et le rendre accessible au plus grand nombre de personnes au moyen du Web.

Pour ce faire, nous avons procuré aux équipes sur le terrain des ordinateurs portables, des appareils photos, des caméras vidéo, des enregistreuses numériques. De nouvelles caméras HD et des microphones sans fil ont été achetés en 2010 à l'aide d'une subvention de la Fondation canadienne pour l'innovation, ce qui a permis d'améliorer considérablement la qualité du son et de l'image des vidéos pour atteindre un niveau professionnel, rendu nécessaire par les exigences accrues des utilisateurs du Web. En effet, les exigences de qualité des images en ligne ont rapidement augmenté entre 2006 et 2010 avec le plus haut débit des bandes passantes permettant la circulation d'images de plus haute définition. Il nous a fallu suivre le mouvement pour rester « compétitifs » et visibles sur le Web, et aussi pour répondre aux exigences accrues des communautés religieuses qui souhaitaient avoir des vidéos de meilleure qualité pour leurs archives et pour la mise en valeur de ce patrimoine immatériel lors de leurs expositions.

L'illustration des textes à l'aide de capsules vidéo, ou « ethno-clips » comme nous les avons nommés, de quelques minutes chacune, a pris de plus en plus d'importance pendant l'inventaire. $\mathrm{Si}$, au départ, on ne réalisait pas de vidéos à chaque entrevue, nous contentant parfois d'un bon enregistrement sonore, nous avons commencé assez rapidement à réaliser systématiquement des vidéos à chaque rencontre et à travailler les montages afin d'illustrer les propos de l'informateur à l'aide de photos historiques et d'extraits vidéo des pratiques. Le patrimoine immatériel, porté par des personnes, est par définition intangible. Par exemple, une description textuelle d'un savoir-faire ne peut avoir la même force ou vivacité qu'une vidéo montrant la pratique commentée par le praticien. La vidéo permet la visualisation directe de l'élément du patrimoine immatériel, l'enregistrement et la présentation du contexte, des expressions, des gestes, des émotions, des sensibilités, bref, du vécu d'une personne ou d'un groupe. Restant fidèle à la tradition du film ethnographique et documentaire, nous avons filmé les informateurs in situ dans leur réalité vécue et les pratiques le plus objectivement possible, sans ajouts de musique et d'autres subterfuges. Les enregistrements sonores et vidéo, généralement de 30 à 90 minutes par entrevue selon le type de récit, ont été réduits au montage en ethno-clips de quatre à cinq minutes, montrant l'essentiel du récit, pour la diffusion sur le Web. Des films plus longs peuvent difficilement être diffusés, car trop longs à télécharger et trop lourds pour la bande passante. De plus, les internautes souhaitent obtenir l'information recherchée très rapidement et ils ne patientraient pas 20 à 30 minutes pour visionner un film ethnographique classique. La tendance actuelle est de réduire encore davantage la durée des films, jusqu'à une et deux minutes. $95 \%$ des 
entrevues ont été filmées. Les archives numériques de l'inventaire comptent 1300 heures d'enregistrement vidéo, 1000 heures d'enregistrements sonores et près de 15000 photographies. Celles-ci sont conservées sur le serveur de la Chaire et en copie sur un serveur de la Faculté des lettres et des sciences humaines par mesure de précaution. Quant au site IPIR, il contient 671 ethnoclips, 680 extraits sonores (4 minutes par fiche en moyenne pour un total de 45 heures), environ 4400 photos et près de 500 photos d'archives.

Pour rendre facilement accessible cette masse de documents multimédia variés, nous avons développé un site convivial, dynamique et interactif. Le premier objectif était de multiplier les voies d'accès à la base de données sur la page d'accueil pour permettre à l'utilisateur de trouver aisément et rapidement l'information recherchée. Nous avons créé sept entrées qui représentent autant de moteurs de recherche : par tradition religieuse (catholique, protestante, juive, orthodoxe, etc.), par type de récits (pratiques, objets, lieux, vies), par communauté, par média (photo, audio, vidéo), par mot-clé, par pratique et par localisation géographique (à l'aide d'une carte interactive). La page d'accueil donne des nouvelles sur le projet IPIR et les activités qui y sont liées. Une bande en haut de la page défile en séquence de cinq secondes des images représentant les cinq principales traditions religieuses du Québec (catholique, protestante, juive, orthodoxe et amérindienne). On y trouve également des onglets qui donnent accès à des informations de base sur l'inventaire : le projet lui-même, les actions culturelles menées dans les communautés, les équipes sur le terrain et les actualités.

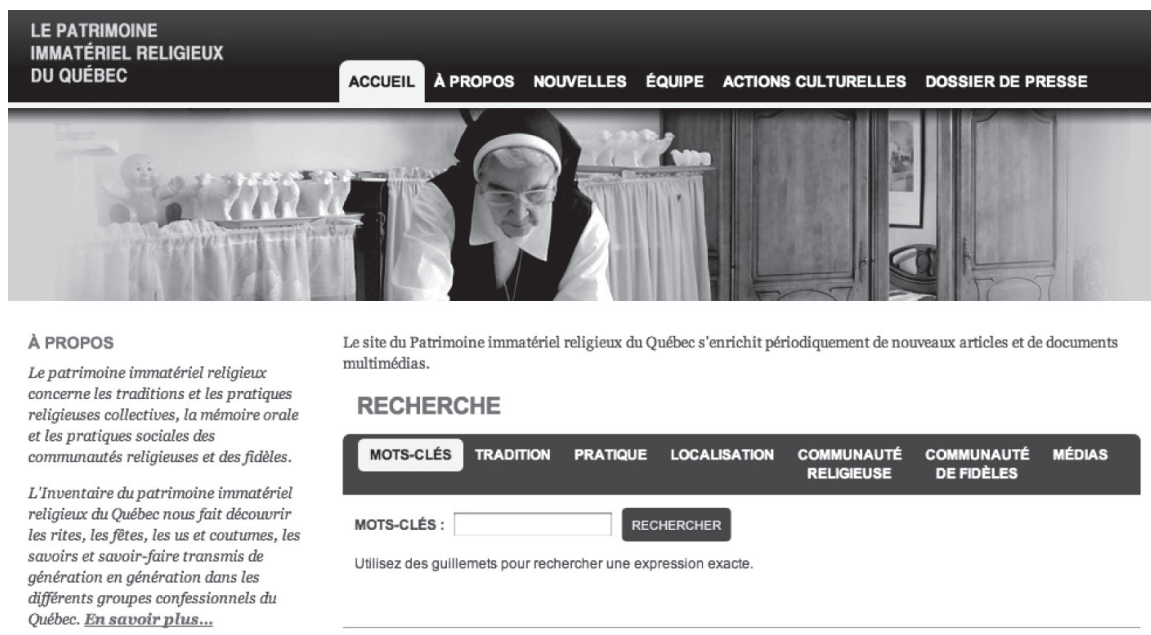

Page d'accueil du site IPIR (www.ipir.ulaval.ca) 
Le second objectif était de permettre à l'utilisateur d'accéder à toute l'information portant sur une pratique à partir d'un même écran ou page, ceci pour éviter la présentation de longues listes et des lectures linéaires. La juxtaposition des quatre médias (description textuelle, extraits sonores, photographies, vidéo) à l'intérieur du même écran permet de donner des informations différentes et complémentaires dans chaque média et de circuler facilement d'un média à l'autre, puis même de les exposer et les étudier côte-à-côte, tout en restant sur la même page. Ce regroupement des principaux médias sur un même écran facilite la narration et la lecture transmédia, c'est-à-dire la migration d'un média à un autre pour obtenir des informations complémentaires sur des supports médiatiques différents ${ }^{46}$. Les fiches traitant d'un même sujet sont liées entre elles par des hyperliens : par exemple, la fiche sur le métier de cuisinière au monastère des Ursulines est liée à la fiche sur le patrimoine alimentaire des Ursulines.

Chacune des fiches contient une très riche documentation ethnographique répartie en trois parties. La première partie est une synthèse sur l'historique de la pratique, de l'objet, du lieu ou de la vie d'une personne, informations issues des récits. La plupart du temps l'historique est narré à la première personne et couvre la période vécue par l'informateur. La deuxième partie présente une description détaillée de la pratique, de l'objet, du lieu ou de la vie de la personne. La troisième et dernière partie est consacrée, d'une part, à l'apprentissage de la pratique par l'informateur (lieux, personnes concernées, modalités) et, d'autre part, à la transmission de sa pratique à d'autres personnes (lieux, personnes concernées, modalités). Toutes ces informations sont d'autant plus riches et précieuses qu'elles n'existaient avant l'inventaire que sous forme orale, n'ayant pas été consignées par écrit.

Nous avons voulu comparer le site de l'IPIR avec d'autres sites semblables dans le monde. La première constatation importante est qu'il n'existe pas d'autres sites sur le patrimoine immatériel religieux ailleurs dans le monde. Toutefois, nous avons identifié quelques sites récents consacrés à la présentation d'inventaires du patrimoine culturel immatériel qui ont fourni des éléments de comparaison éclairants. Le site de l'inventaire de la France, comprenant 231 entrées, contient de très riches descriptions ethnographiques (d'ailleurs souvent plus élaborées que dans l'IPIR) et des photos, mais pas de vidéos. Il est organisé sous forme de liste, sans moteur de recherche. De même, celui de la Suisse, composé de 167 entrées, possède des descriptions

46. Cette notion de narration transmédia (traduction libre de l'expression anglaise transmedia storytelling) a été développée par un chercheur américain, professeur au Massachussetts Institute of Technology (Міт) à Boston. Voir : Henry Jenkins, "Transmedia Storytelling », Technology Review, 2003. Retrieved December 2, 2010 ; et Henry Jenkins, «Transmedia 202 : Further Reflections » Confessions of an AcaFan. 2011. 
de pratiques (plus sommaires, soit un paragraphe seulement) et des photos, mais très peu de vidéos. Comprenant quelque 152 entrées, le site de l'inventaire du patrimoine immatériel de l'Écosse est présenté sous forme d'un wiki. Comme c'est souvent le cas avec les wikis, les informations ethnographiques des entrées sont de qualité inégale et parfois très succinctes (seulement deux à trois lignes de texte). On y trouve souvent des liens vers d'autres sites, mais très peu de photos et de vidéos (les quelques vidéos présentées étaient accessibles via un lien vers Youtube). L'inventaire wiki de l'Écosse démontre une fois de plus que l'approche très participative peut être une plus-value au niveau de la mobilisation des acteurs, mais produit un très faible rendement au niveau de l'utilisation. La Belgique francophone (Wallonie/Bruxelles) a rassemblé sur un site certaines pratiques de l'inventaire du patrimoine immatériel qui comprend une liste de 31 fiches présentées à l'aide de textes, de photos et d'une carte pour localiser la pratique, à la manière d'un magazine culturel ou d'une encyclopédie ${ }^{47}$. Chaque fiche contient également une vidéo de deux minutes qui fait état de la pratique de manière artistique comportant un dialogue entre deux narrateurs sur fond de musique. Certaines fiches contiennent une deuxième vidéo plus longue, généralement de quatre à six minutes, nommée « interview » qui est, comme son nom l'indique, une entrevue de type ethnographique avec deux, trois, quatre et parfois cinq praticiens. L'idée d'avoir deux vidéos, l'une destinée au grand public et l'autre conçue davantage pour les spécialistes, est intéressante. Composée de trois à quatre pages, chaque fiche est divisée en huit rubriques qui répondent aux questions suivantes : c'est quoi ? (description), ça se passe où ? (lieu), c'est quand ? (calendrier), un brin d'évasion (comparaison avec une pratique semblable ailleurs dans le monde), un brin d'histoire (historique de la pratique), un brin de poésie (poétique de la pratique), un abécédaire (glossaire) et les liens vers d'autres sites. Les fiches du site ne contiennent pas d'informations sur la transmission, ni sur l'état de conservation de la pratique, ni sur les mesures de sa sauvegarde. Il y a à la fin de la fiche des hyperliens vers d'autres sites belges ou étrangers. Ainsi, le site se rapproche plus d'une encyclopédie multimédia que d'un inventaire du patrimoine culturel immatériel de type ethnographique. La Belgique flamande possède un site de 40 éléments présentés sous forme de texte court (un paragraphe), de photos et de quelques vidéos. Le site est aussi muni d'hyperliens qui mènent vers d'autres éléments et une carte qui permet de localiser la pratique. Cependant, on ne peut accéder à la banque de données que par mot clé et le nom de la pratique. Le site de l'inventaire de l'Inde comprend texte, photos et vidéos, mais il est présenté sous forme d'une liste de pratiques ${ }^{48}$. Il est extrêmement difficile d'y naviguer en raison

47. www.patrimoinevivantwalloniebruxelles.be/patrimoines/pratiques/fiche_pratiques/?n=41.

48. Voir inventaire du PCI de l'Inde : ignca.nic.in/ich_inventory.htm. 
de l'absence de moteurs de recherche, mais aussi parce que les photos et les vidéos ne sont pas compressées et difficiles à ouvrir. À la suite de ce tour d'horizon, force est de constater que le site IPIR se compare très favorablement en ce qui concerne la qualité des contenus, la variété des médias et la facilité de navigation.

Cependant, il pourrait être amélioré sur deux plans : d'une part, par une meilleure qualité de vidéos et, d'autre part, par l'ajout d'une évaluation de la viabilité de la pratique et, éventuellement, d'un plan de sauvegarde si la pratique est très menacée. Sur le plan technique, il est opportun d'accroître toujours la qualité des vidéos au niveau de la prise d'images avec des caméras professionnelles (ex: Sony HDRFX 1000 à cassette, Canon 5D, Panasonic HPX370) et des montages encore plus élaborés et courts (2 à 3 minutes) réalisés par un vidéaste professionnel. Il faut être en mesure de répondre aux exigences toujours plus élevées des utilisateurs du Web et posséder de bonnes images afin de pouvoir les utiliser et les valoriser dans d'autres outils multimédia, comme des applications mobiles et des expositions virtuelles en ligne. Dans la mesure où il est relativement coûteux de préparer un terrain ethnographique et un film (prises de contacts, organisation de rendez-vous, frais de déplacement et de séjour des équipes, achat d'équipements de qualité professionnelle), il vaut mieux investir dans la qualité pour assurer des retombées certaines et des usages multiples du produit. Le retour sur l'investissement ne peut qu'être meilleur. Sur le plan du contenu, il serait très important d'ajouter deux autres rubriques à la fiche : l'une décrivant la viabilité de la pratique et, dans le cas où elle est menacée, une autre présentant des mesures de sauvegarde. L'inventaire de la France pourrait servir de modèle : sa fiche reprend les catégories de la fiche d'inventaire du Québec et, s'inspirant des recommandations de l'UNESCO, elle inclut ces deux catégories supplémentaires. C'est pendant l'inventorisation sur le terrain que la viabilité de la pratique peut être le mieux évaluée et des mesures de sauvegarde envisagées en collaboration avec les praticiens.

\section{Analyse de la fréquentation du site IPIR}

L'analyse de la fréquentation du site IPIR nous permet d'appréhender directement l'intérêt que représente l'inventaire du patrimoine immatériel religieux et de mesurer ses retombées de manière quantitative. À la suite du lancement du site IPIR en mai 2010, nous y avons fait installer (en août 2010) un compteur Google Analytics afin de recueillir des informations précises sur le nombre de visiteurs, la durée de leurs visites, leur provenance, leur âge et leurs habitudes de consultation. Dans la mesure du possible, nous avons comparé ces données avec celles d'autres sites d'inventaires du patrimoine immatériel. 
À l'étude des données, ce qui frappe tout d'abord c'est la forte fréquentation du site. Depuis l'installation du compteur à l'été 2010 jusqu'à l'été 2014, soit le moment de réalisation de la présente étude, le site a été consulté 252775 fois (nombre de sessions ouvertes) et a accueilli 200231 utilisateurs, soit des visiteurs qui ont consulté des pages du site. Il s'agit de plus de 50000 utilisateurs par année en moyenne ou de 4350 utilisateurs par mois, un chiffre relativement élevé compte tenu du fait que l'information du site est très spécialisée - un inventaire du patrimoine immatériel religieux et exclusivement en langue française. Il est intéressant aussi de noter que la fréquentation du site augmente de manière assez marquée depuis son ouverture. Pendant les deux premières années, il y a eu 51000 utilisateurs, alors qu'il y en a eu 149224 au cours des deux dernières années, soit trois fois plus! Sur une base journalière, la fréquentation du site montre de fortes pointes d'utilisation pendant la période de Pâques. Pâques est le temps fort du calendrier chrétien; il n'est donc pas étonnant que le site soit très fréquenté à cette période de l'année. Les gestionnaires du site reçoivent alors des demandes par courriel ou par téléphone. Si l'on en juge d'après ces demandes faites à la suite d'une prise de contact avec le site, on constate que le site est fortement sollicité alors par des élèves, des étudiants et des professeurs qui conduisent des recherches sur des sujets liés aux fêtes de Pâques, mais aussi des journalistes à la recherche d'informations pour la préparation d'articles ou d'émissions de radio et de télévision. La durée moyenne des sessions est d'environ deux minutes, le nombre de pages consulté par session est de 1,83, ce qui se situe dans la moyenne des sites de ce type.

La fréquentation de l'IPIR est bien supérieure à celle des sites d'inventaires comparables pour lesquels nous avons pu obtenir des informations. Le site d'inventaire du patrimoine immatériel de la Belgique flamande accueille environ 20200 visiteurs (sessions) et 14500 utilisateurs par année qui visionnent 2,51 pages par visite ${ }^{49}$, soit trois fois moins qu'IPIR. Il faut dire que ce site est assez récent et présenté uniquement en langue flamande, ce qui ne favorise pas son accès. En revanche, l'inventaire de l'Écosse n'a reçu que quelque 36481 utilisateurs depuis sa mise en ligne en avril 2011, soit environ 12160 utilisateurs par année ${ }^{50}$, moins encore que le site de la Belgique flamande, alors qu'il est en langue anglaise. Le cas de l'Écosse démontre bien que la langue de présentation n'est pas le seul facteur qui agit sur la fréquentation. Il est possible de l'expliquer par le fait que ce site est sous forme d'un wiki incomplet et peu convivial. Favorisant l'approche très participative, dans la mesure où chacun y dépose ses textes, le wiki laisse souvent à désirer en ce qui concerne la qualité des textes et des images. À titre de comparaison avec

49 Ellen Janssens, Hans van der Linden et Bram Wiercx, op. cit., p. 98.

50. www.ichscotlandwiki.org/index.php?title=Welcome 
un autre site québécois, le site d'Inventaire des ressources ethnologiques du patrimoine immatériel (IREPI) reçoit environ 20000 visiteurs en moyenne par année depuis l'installation du compteur à l'été 2010, soit environ $25 \%$ de plus que celui de la Belgique flamande. C'est dire qu'IpIR, site québécois du patrimoine immatériel religieux, reçoit deux fois et demi plus d'utilisateurs qu'IrEPI, site québécois du patrimoine immatériel laïc. Il nous a été impossible d'obtenir d'autres données de fréquentation, soit parce que les sites n'ont pas de compteurs (c'est le cas du site de la France et de celui de la Suisse), soit que l'information sur la fréquentation n'est pas accessible ${ }^{51}$. Quoi qu'il en soit, la comparaison de la fréquentation de ces trois cas (Belgique flamande, Écosse, Québec-IREPI) avec celle de l'IPIR montre bien que ce dernier connaît une utilisation deux à trois fois plus élevée, ce qui est significatif.

Les utilisateurs du site IPIR sont relativement jeunes et composés majoritairement d'hommes. Un peu plus du quart (27,5\%) est âgé entre 18 et 24 ans. Lorsque l'on ajoute la catégorie d'âge entre 25 et 34 ans, les moins de 34 ans regroupent $61 \%$ des utilisateurs. Contrairement à ce que l'on pourrait penser, les catégories de personnes plus âgées, ayant fréquenté les églises et étant influencées dans leur jeunesse par la pratique religieuse, utilisent peu le site ( $11 \%$ pour les 55 ans et plus), soit cinq fois moins. Il est vrai qu'il faudrait pondérer quelque peu ces chiffres dans la mesure où les moins de 34 ans utilisent plus régulièrement Internet que les catégories d'âge de plus de 55 ans. Mais cette pondération conduit à multiplier par deux, tout au plus. En effet, le CEFrIo, organisme financé par le ministère de l'Économie, de l'innovation et des exportations du Québec, qui mène régulièrement des enquêtes sur les utilisations du Web sur tout le territoire québécois depuis plus de 12 ans, constate une nette progression chez les 55 à 74 ans au cours des dernières années. En 2012, $73 \%$ des 55 à 64 ans et $56 \%$ des 65 à 74 ans en étaient des utilisateurs réguliers, contre $95 \%$ pour les catégories de 18 à 34 ans $^{52}$. On voit qu' aujourd'hui la différence entre les utilisateurs jeunes et les utilisateurs plus âgés, soit de la génération d'après-guerre, n'est plus très importante. Le grand écart constaté entre les 18-34 ans et les 55-74 ans dans la fréquentation du site IPIR ne tient donc pas à des tendances intergénérationnelles d'utilisation du Web. Tout indique une forte attraction des jeunes pour le patrimoine religieux, ce qui semble annoncer une tendance nouvelle. Visiblement, le site est très sollicité par les étudiants du cégep et ceux de l'Université, et par les professeurs. Par ailleurs, nous savons que les professeurs du cours d'Éthique et culture religieuses s'en servent dans

51. J'ai essayé d'obtenir des données sur la fréquentation des sites d'inventaires de Wallonie/ Bruxelles, de l'Inde, de la Chine et du Brésil, mais sans succès.

52. Voir le site Netendances du Cefrio : http://www.cefrio.qc.ca/projets-recherches-enquetes/ intervention-citoyenne-services-publics/netendances. 
leurs cours, ce qui contribue à expliquer cette assez forte fréquentation par les jeunes. Le site est utilisé majoritairement par les hommes (54\%), ce qui surprend un peu dans la mesure où la vaste majorité des récits inventoriés portent sur des congrégations religieuses féminines (70\%).

Le site IPIR connaît une grande distribution géographique puisque les utilisateurs viennent de 174 pays dans le monde. Il n'en demeure pas moins que les utilisateurs proviennent en majorité du Canada francophone (64\%), suivi de la France (22\%), et des États-Unis (3,7\%). Les autres utilisateurs sont issus en grande partie de pays francophones européens (Belgique, Suisse), africains (Côte d'Ivoire, Cameroun) et nord-africains (Maroc, Algérie, Tunisie). En faisant une ventilation par villes du Québec, on constate que la majorité des utilisateurs sont de Québec (46\%) plus que de Montréal (36\%), la métropole qui regroupe pourtant près de la moitié de la population de la province. Cet écart s'explique sûrement par le fait que le projet IPIR a été mené par des chercheurs de l'Université Laval à Québec et que le site est davantage connu dans la capitale nationale.

Comment expliquer cette fréquentation relativement forte de l'IPIR au Québec et dans le monde, par rapport à d'autres sites d'inventaires comparables ? Outre la grande taille, la qualité du contenu du site et la facilité de la navigation, questions que nous avons précédemment abordées, l'IPIR traite d'un patrimoine dont l'intérêt est grandissant. En effet, la thématique du patrimoine religieux suscite de plus en plus d'intérêt au Québec, mais aussi ailleurs dans le monde. Compte tenu de ce contexte, il n'est sans doute pas étonnant qu'un site dédié spécialement au patrimoine immatériel religieux attire plus que les sites d'inventaire qui portent seulement sur le patrimoine laïc.

\section{Retombées dans les communautés religieuses et sauvegardes effectives}

Pour mener à bien le bilan, évaluer les retombées et envisager les perspectives d'avenir, il nous a paru essentiel d'interroger les informateurs qui ont participé à ce grand projet, quelques années après leur collaboration, pour connaître leur perception et les usages effectifs et possibles des archives produites par les ethnologues - un témoignage précieux - qui leur ont été remises. Nous estimons cette démarche d'évaluation, plus qualitative, faite par les communautés elles-mêmes, importante et indispensable pour mener un bilan rétrospectif, et permettre l'analyse critique des retombées et perspectives d'avenir de l'inventaire du patrimoine immatériel religieux. Les témoignages recueillis démontrent la pertinence et l'impact de l'inventaire IPIR pour les communautés, le vaste potentiel d'exploitation des données, la multitude de retombées réelles et mesurables dans tous les secteurs de sauvegarde du 
patrimoine immatériel, soit en termes de mise en valeur, de documentation, de sensibilisation et de transmission.

\section{Méthodologie}

Dans un premier temps, nous avons commencé par sélectionner une vingtaine de paroisses et communautés parmi celles qui ont collaboré à l'inventaire. La liste des communautés et paroisses contactées était représentative dans son ensemble de la répartition des participants qui ont collaboré à l'IPIR (confessions, types de communauté, communautés culturelles et répartition géographique). Une liste de questions ouvertes a été réalisée. Ces questions portaient sur les archives produites par l'IPIR, leur utilisation présente et future, les perceptions et l'utilité de l'inventaire, ainsi que sur la sensibilisation au patrimoine immatériel et les mesures de sauvegarde qui pourraient être envisagées. L'entrevue avait un double objectif : s'informer de l'impact de la collaboration à l'inventaire sur les mesures de reconnaissance et de sauvegarde de la communauté ou de la paroisse sur son patrimoine immatériel, et relancer l'intérêt de celles-ci pour l'utilisation de ses archives produites par l'inventaire. Au total, 14 entretiens téléphoniques ont été réalisés. Ceux-ci ont duré entre 25 et 45 minutes.

Les communautés rencontrées sont réparties à travers le Québec. Elles proviennent de dix régions administratives différentes : Montréal, Québec, Outaouais, Abitibi-Témiscamingue, Montérégie, Centre-du-Québec, ChaudièreAppalaches, Charlevoix, Saguenay-Lac-Saint-Jean et Bas-Saint-Laurent. Les types de communautés sont bien représentés, entre les communautés de frères et de sœurs, et les paroisses. Un souci de représentativité des communautés et paroisses dans la société québécoise a été observé. Ainsi, on retrouve en majorité des communautés catholiques, mais sont aussi représentées les communautés orthodoxes, protestantes, juives et musulmanes. La grande majorité des entrevues se sont faites avec des personnes québécoises de souche, mais nous avons aussi rencontré des représentants de communautés culturelles (italiennes, espagnoles, russes, maghrébines et amérindiennes) ${ }^{53}$.

Nous avons tenté de joindre la personne-ressource initiale avec laquelle nous avions collaboré lors de l'inventorisation. Parfois celle-ci avait changé

53. Liste des communautés rencontrées : Paroisse Notre-Dame-de-Saint-Roch (catholique, Québec) ; Communauté des Antoniennes de Marie (catholique, Chicoutimi) ; Communauté des petites Franciscaines de Marie (catholique, Baie-Saint-Paul) ; Communauté des Cisterciens de Rougemont (catholique, Rougemont) ; Communauté des sœurs de Notre-Dame-du-Rosaire (catholique, Rimouski); Communauté des Cisterciennes de N.-D.-du-Bon-Conseil (catholique, Saint-Benoît-Labre) ; Cathédrale des Saints-Pierre-et-Paul (orthodoxe, Montréal) ; Saint Andrew Prebyterian Church (protestant, Québec) ; Communauté des frères mineurs capucins (catholique, Montréal) ; Église Sainte-Clotilde (catholique, Kitcisakik) ; Communauté des frères du Sacré-Cœur (catholique, Victoriaville) ; Centre soufi Naqshbanti (musulman, Montréal) ; Communauté des sœurs de la charité d'Ottawa (catholique, Gatineau); Spanish and Portugese Synagogue (juive, Montréal). 
de poste et avait été déplacée dans une autre communauté (3), ou encore elle était absente (2). Cinq des quatorze personnes-ressources contactées n'étaient plus les mêmes qu'au moment de l'inventaire. Toutefois, la plupart avaient été informés par leurs prédécesseurs et étaient au courant du projet.

\section{Mise en valeur du patrimoine immatériel et de l'inventaire par les communautés elles-mêmes}

L'inventaire a fortement contribué au renforcement des capacités d'une communauté à mettre en valeur les éléments de son patrimoine immatériel. L'ensemble des communautés a été informé des résultats de l'inventaire par une présentation offerte par les ethnologues, les données ont été validées par les communautés, restituées, puis les fiches d'inventaire ont servi à la réalisation de nombreux projets de mise en valeur par les communautés elles-mêmes suite au passage des ethnologues chez elles. Ces projets ont utilisé comme supports les fiches de l'inventaire pour informer les membres des communautés ou la population du travail accompli, comme il a été mentionné. Selon Chiara Bortolotto, le retour des données aux communautés, tel qu'effectué par les inventaires IREPI et IPIR, est l'une de ses principales forces, car elle permet la réappropriation des données par les communautés et ainsi leur mise en valeur : "L'implication des chercheurs sur le terrain et leurs efforts pour permettre aux communautés de "se réapproprier les pratiques" sont présentés comme des formes de participation, terme clé des politiques de sauvegarde du $\mathrm{P}_{\mathrm{CI}}$ : observation participante, retour à la communauté et accessibilité des données », ce qui constitue «l'approche novatrice [...] par rapport aux inventaires traditionnels tournés vers la documentation scientifique ${ }^{54}$. » L'inclusion d'un élément dans l'inventaire IPIR, et le processus participatif qui le précède en amont et qui lui succède, permettent de « faciliter la participation des communautés locales au processus de capitalisation par la réappropriation de pratiques considérées par leur inclusion dans un inventaire ${ }^{55} »$.

L'utilité de l'inventaire a été, selon les communautés, de permettre la mise en valeur des pratiques et savoir-faire d'un patrimoine méconnu. Ainsi, à la question « Comment qualifieriez-vous l'utilité de l'inventaire : petite, moyenne, grande, très grande ? », les Antoniennes de Marie ont répondu : « Très grande, car l'inventaire a su mettre le patrimoine de sœurs qui travaillaient dans l'ombre en valeur ${ }^{56}{ }_{\eta}$. De même, les responsables de l'église presbytérienne Saint Andrew à Québec ont qualifié l'inventaire de «très

54 Chiara Bortolotto, « The French Inventory of Intangible Cultural Heritage », op. cit., p. 162.

55. Op. cit., p. 160.

56. Entrevue avec Ginette Laurendeau, des Antoniennes de Marie, Chicoutimi, le 26 novembre 2013. 
important ${ }^{57} »$, alors que les Cisterciennes de Notre-Dame-du-Bon-Conseil de Saint-Benoît-Labre (Chaudière-Appalaches) l'ont jugé « très utile ${ }^{58}$ ».

Les archives de l'inventaire ont été utilisées activement par huit communautés de l'échantillon, selon les personnes-ressources des neuf communautés où nous avons pu rejoindre la personne-ressource de l'inventaire. Les données ont servi tant à des projets de valorisation destinés à la communauté qu'au grand public. Sept paroisses et communautés ont prévu d'utiliser les données de l'inventaire au cours de l'année 2014-2015. Deux d'entre elles utilisent les vidéos de l'IPIR pour faire des présentations à l'extérieur de la communauté, pour les aînés de la municipalité dans un cas, et pour des organismes parapublics en lien avec la communauté dans le but de la faire connaître, dans un second cas. Une troisième a utilisé plusieurs photos et vidéos de l'inventaire IPIR pour alimenter son site et son blogue et pour réaliser une exposition dans le cadre de l'anniversaire de la congrégation. Une quatrième communauté s'est, quant à elle, appropriée la vidéo de présentation préparée par les ethnologues pour la projeter dans la paroisse, en ajoutant une présentation narrative du curé.

Le support vidéo est l'un des moyens qui a été le plus utilisé. À la paroisse Notre-Dame-de-Saint-Roch à Québec, une vidéo a été réalisée par la communauté pour exposer différents éléments du patrimoine immatériel religieux inventoriés. La vidéo est visible à la paroisse. Une exposition, intitulée «Bénis de Dieu : récits des communautés religieuses du Québec », a également été créée sur le thème du patrimoine immatériel religieux à partir des entrevues recueillies par les ethnologues pour l'inventaire IPIR, et présentée au moyen des technologies multimédia (panophotographie, son multicanal, vidéo), dont il a été question plus haut. Rappelons que les données de l'inventaire ont aussi servi à alimenter le site de la communauté des Cisterciens de Rougemont en photos et autres informations. Pour le $75^{\mathrm{e}}$ anniversaire de la communauté, plusieurs documents de l'IPIR ont été utilisés par la communauté pour faire une exposition à l'aide d'un ordinateur. Les archives ont également servi à des présentations de la communauté aux aînés des paroisses, mais aussi aux aînés de la ville et à des religieuses dans trois communautés interrogées.

\section{Utilisation future des archives et de l'inventaire IPIR}

Le retour auprès des communautés visait non seulement à connaitre l'utilité de l'inventaire pour celles-ci, mais a permis également de s'informer sur leurs projets à venir en lien avec l'inventaire et ainsi d'évaluer leur intérêt pour le

57. Entrevue avec Katherin Burgess, de l'église presbytérienne Saint Andrews, le 12 décembre 2013.

58. Entrevue avec Marie Saint-Pierre, des Cisterciennes de Notre-Dame-du-Bon-Conseil de Saint-Benoît-Labre (Chaudière-Appalaches), le 15 décembre 2013. 
patrimoine immatériel et pour les archives réalisées par les ethnologues dont elles disposent maintenant. Les entretiens ont également permis de relancer avec ferveur l'intérêt des communautés.

Sept paroisses et communautés ont prévu utiliser les données de l'inventaire au cours des deux prochaines années, 2014 et 2015 : deux souhaitent réaliser une vidéo de présentation de la communauté pour la faire connaître à l'extérieur, une troisième souhaite utiliser les données textuelles et audiovisuelles pour créer un espace muséal, une quatrième et une cinquième pour alimenter le site de la communauté ou un réseau de documentation plus large, on projette de réaliser dans une sixième une activité de mise en valeur du patrimoine immatériel (la forme du projet est toutefois imprécise), ainsi qu'une recherche historique dans une septième. Dans quatre cas, les communautés n'ont pas envisagé de projets particuliers puisqu'elles se disent satisfaites du travail qui a été réalisé, ces archives permettant de laisser un témoignage et une trace dans l'histoire.

\section{IPIR : la sauvegarde par l'archivage de la mémoire vivante et la documentation}

La fonction de sauvegarde de l'inventaire a été soulignée par l'ensemble des communautés, conscientes de leur fragilité. L'inventaire a permis de sauvegarder une mémoire menacée de disparition par la collecte des récits oraux et leur diffusion. Les Antoniennes de Marie étaient désireuses de participer à l'inventaire dans cet objectif, « elles y tenaient beaucoup, car ces documents étaient précieux en sachant que les sœurs avaient une courte espérance de $v^{5 i}{ }^{59}$ ». Pour la communauté juive de Montréal, l'inventaire a été « Très utile, oui, parce qu'on perd des générations. Vous nous avez aidé à sauver $1^{\prime}$ histoire ${ }^{60} \gg$. De même, sœur Marie Saint-Pierre des Cisterciennes de NotreDame-du-Bon-Conseil de Saint-Benoît-Labre (Chaudière-Appalaches) fait bien comprendre que les archives témoigneront de leur œuvre :

Si la communauté disparaît, ça ne change rien, car c'est une autre culture religieuse (on parle ici d'une communauté contemplative), une autre beauté, une tradition lointaine qui est documentée. Nous sommes sensibles à ce que nous vivons, nous avons nos archives écrites et orales et ce sera récupéré. L'Église prendra soin de ce que nous avons. Des bâtiments disparaîtront, mais pas notre œuvre. ${ }^{61}$

59. Entrevue avec Ginette Laurendeau, des Antoniennes de Marie, Chicoutimi, le 26 novembre 2013.

60. Entrevue avec Schachar Orenstein, de la Spanish \& Portugese Synagogue de Montréal, le 17 décembre 2013.

61. Entrevue avec sœur Marie Saint-Pierre, des Cisterciennes de Notre-Dame-du-Bon-Conseil de Saint-Benoît-Labre (Chaudière-Appalaches), le 15 décembre 2013. 
Le frère Paul Myles de la congrégation des frères du Sacré-Cœur à Victoriaville tient à peu près le même discours, mais présenté en d'autres termes :

La philosophie de la communauté est que, selon moi, ce qui est pertinent passera à l'histoire. Il faut aller dans le sens de la société, et non rester continuellement comme cela a toujours été, en s'accrochant à une époque, sinon on se referme sur nous-mêmes. En s'ouvrant au changement, en suivant les jeunes, c'est là qu'il y a de l'avenir, et une volonté de prise en charge de l'œuvre. [...] On garde ainsi une trace pour les familles, les confrères, et la ville parce que la communauté a participé à sa fondation. [...] Ce qui est important est de laisser des traces de l'histoire pour la population et l'avenir. ${ }^{62}$

Ces communautés semblent avoir une conception résignée face aux transformations de la société : elles acceptent que leur patrimoine disparaisse avec elles si la société québécoise ne s'y intéresse plus. La trace laissée par les enseignements - et les témoignages tels que ceux recueillis par l'IPIR demeureront pour le futur. Ils sont garants de la connaissance et de la mémoire des savoir-faire de ces communautés. C'est pour elles l'utilité principale de l'inventaire.

La fonction de documentation a été soulignée par de nombreuses communautés. Le plus souvent, ce sont les photos et vidéos qui sont utilisées comme support, ainsi dans la paroisse protestante Saint Andrew, chez les Cisterciens ou encore la communauté Soufie. Des projets d'exposition sont le plus envisagés ou évoqués comme des moyens d'utiliser les ressources. L'inventaire peut aussi servir de référence documentaire pour la réalisation de demandes de reconnaissance; ainsi à la Fondation Saint-Roch, l'inventaire IPIR pourrait être une aide documentaire importante pour réaliser « un projet de reconnaissance de la paroisse en tant que sanctuaire de la miséricorde ${ }^{63} \gg$. L'Église Sainte-Clotilde (catholique, Kitcisakik) souhaite utiliser les données de l'inventaire pour le $75^{\mathrm{e}}$ anniversaire du diocèse d'Amos afin de retracer l'historique de la communauté de façon plus vivante ; on veut organiser une exposition d'objets liturgiques, en misant sur le patrimoine immatériel, dans la cathédrale d'Amos, en utilisant les portes en verre de la sacristie comme des vitrines muséales ${ }^{64}$. La communauté orthodoxe de la cathédrale des SaintsPierre-et-Paul (Montréal) souhaiterait réaliser une publication qui présente l'histoire, la mission et les valeurs de la communauté, en utilisant les données de l'inventaire IPIR ${ }^{65}$.

62. Entrevue avec le frère Paul Myles, de la congrégation des frères du Sacré-Cœur à Victoriaville, le 10 décembre 2013.

63. Entrevue avec Réal Grenier, curé, de l'église Saint-Roch à Québec, le 9 décembre 2013.

64. Entrevue avec sœur Renelle Lassalle, de l'église Sainte-Clotilde à Kitcisakik (AbitibiTémiscamingue), le 17 décembre 2013.

65. Entrevue avec Anatoly Melnik, le 15 décembre 2013. 


\section{IPIR : un outil pour enrichir la compréhension du patrimoine matériel}

L'inventaire a permis de créer un lien inédit et extrêmement utile avec le patrimoine matériel religieux et d'accroître ainsi sa compréhension. Il a aussi permis de sensibiliser les communautés à ce lien entre deux types de patrimoines souvent séparés. Suite au projet IPIR, le projet Patrimoine in situ, dirigé par la Société des musées québécois, a réalisé un inventaire des biens religieux de valeur en 2009 dans la communauté des Antoniennes; près de 320 objets sont documentés en ligne, et on en retrouve beaucoup à présent au musée du couvent, le Musée des sœurs Antoniennes de Marie, qui s'intéresse principalement à l'histoire et au patrimoine matériel. Lors de ce deuxième inventaire, il a été plus facile de sélectionner le matériel, car les religieuses avaient été sensibilisées à leur patrimoine ${ }^{66}$.

Les fiches et données de l'inventaire IPIR sont venues compléter le contenu des expositions sur le patrimoine matériel des communautés religieuses. Auparavant, elles n'avaient de documentation que sur cette catégorie de patrimoine. Les expositions traitaient donc la plupart du temps des objets sacrés des communautés, du mobilier ou de l'architecture des bâtiments, tel qu'à l'église Saint-Roch de Québec ou encore dans le musée des Franciscaines. Les communautés ont donc accueilli très favorablement cette initiative et ont intégré les données relatives à leur patrimoine culturel immatériel dans ces expositions. La communauté des sœurs de Notre-Dame-du-Rosaire a utilisé les données de l'inventaire pour enrichir le contenu « sur l'histoire de la communauté dans leur Musée ${ }^{67} \gg$. Un espace muséal a ouvert en 2006 chez les Franciscaines dans le but de transmettre des éléments de la communauté religieuse. Grâce à l'inventaire, elles peuvent plus facilement communiquer leurs valeurs et leur mission, en vue de les transmettre aux associés laïcs pour qu'à leur tour ils transmettent ce qu'on leur a enseigné.

L'église Sainte-Clotilde (catholique, Kitcisakik) souhaite associer le patrimoine matériel et immatériel de la communauté et le mettre en valeur de façon intégrée. Pour fêter le $75^{\mathrm{e}}$ anniversaire du diocèse, une exposition d'objets liturgiques mettra en valeur le patrimoine immatériel de la communauté dans la cathédrale d'Amos ${ }^{68}$. La combinaison des deux patrimoines, matériel et immatériel, offre une nouvelle perspective, plus riche et complète du patrimoine religieux, qui permet d'éclairer la connaissance de ses significations et de développer son interprétation.

66. Entrevue avec sœur Ginette Laurendeau, des Antoniennes de Marie à Chicoutimi, le 26 novembre 2013.

67. Entrevue avec sœur Auréla Cyr, de la congrégation des sœurs de Notre-Dame-du-SaintRosaire à Rimouski, le 28 novembre 2013.

68. Entrevue avec sœur Renelle Lassalle, de l'église Sainte-Clotilde à Kitcisakik (AbitibiTémiscamingue), le 17 décembre 2013. 
La collaboration menée entre la Chaire de recherche du Canada en patrimoine ethnologique de l'Université Laval et la Société des musées québécois (SMQ) a permis d'enrichir la compréhension du mobilier religieux, ses usages et ses significations, par l'ajout d'une catégorie relative au patrimoine culturel immatériel. Un onglet patrimoine culturel immatériel figure désormais dans l'inventaire du mobilier mené par la SMQ.

\section{IPIR : un outil de sensibilisation au patrimoine immatériel et de transmission}

La sensibilisation au patrimoine immatériel religieux est assez récente dans les communautés religieuses, et plusieurs ne connaissaient pas ou très peu la notion de " patrimoine immatériel » avant leur participation au projet d'inventaire. L'inventaire a donc joué un rôle très important d'information et de sensibilisation à la connaissance de cette notion et à l'importance de la sauvegarde. Il a aussi servi d'outil de transmission aux plus jeunes ainsi qu'à l'ensemble de la communauté. Notons que quatre personnes interrogées n'étaient pas en charge du projet et ne pouvaient donc pas réellement en témoigner. En excluant ces dernières, la collaboration avec l'IPIR a sensibilisé au moins dix des quatorze paroisses et communautés rencontrées à la richesse de leurs traditions et de leur savoir-faire, comme en témoigne une religieuse des Antoniennes de Marie :

Nous avons été sensibilisées à la richesse de notre patrimoine grâce à l'intérêt porté par des gens de l'extérieur, tels que les ethnologues du projet IPIR. Le travail n'avait rien d'extraordinaire et, pour les sœurs, les tâches étaient « naturelles » et elles y travaillaient avec humilité. Avec l'intérêt porté sur leurs savoirs, elles ont compris que c'était précieux et cela leur a permis de le partager, alors qu'elles sont habituellement assez réservées. En sachant que certaines sont les seules aujourd'hui à posséder un savoir-faire, et que certaines ne sont plus capables d'en faire aujourd'hui, le projet IPIR est arrivé au tout dernier moment. Beaucoup sont aujourd'hui décédées et l'IPIR a pu conserver et partager la mémoire. ${ }^{69}$

Ce témoignage de sœur Ginette Laurendeau des Antoniennes de Marie nous livre en quelques mots toute l'importance et les fonctions de l'inventorisation, en tant que moyen de sensibilisation, d'identification et de connaissance du patrimoine immatériel, d'une part, et, d'autre part en tant que moyen de sauvegarde d'un patrimoine très fragile, sinon déjà disparu, et de transmission.

Les données de l'inventaire ont été consultées, à fréquence variable selon les communautés et selon leur âge. La sensibilisation a été importante pour informer les plus jeunes des communautés. Ainsi, chez les Cisterciens, ces derniers connaissent moins l'histoire de la communauté. « Cela a permis

69. Entrevue avec sœur Ginette Laurendeau, des Antoniennes de Marie à Chicoutimi, le 26 novembre 2013. 
de les sensibiliser à la richesse de leur patrimoine ${ }^{70} »$. Chez les sœurs de la Charité d'Ottawa, l'inventaire a aussi servi au renforcement des capacités de sauvegarde du patrimoine culturel immatériel. Il a suscité une prise de conscience et a stimulé les sœurs pour la sauvegarde de certains éléments de leur patrimoine : "L'éveil s'est fait chez les francophones et il y a eu des bénéfices : pour les cadrans solaires, la sœur qui travaille au musée s'est donné la peine et on la sent plus intéressée ${ }^{71}$. »

L'inventaire a sensibilisé les citoyens également, car des personnes de l'extérieur demandent des informations complémentaires sur la communauté des sœurs du Rosaire, ainsi que des chercheurs, selon quatre autres communautés qui ont reçu des demandes de renseignement au sujet de plusieurs fiches de l'inventaire. Régulièrement, la communauté des Cisterciens de Rougemont est contactée par des personnes qui ont visité le site IPIR et qui souhaitent obtenir des informations complémentaires sur des éléments du patrimoine qui apparaissent sur le site ${ }^{72}$.

\section{Le rôle du Web}

Ce rôle a été souligné à plusieurs reprises pour la sensibilisation, la transmission et la valorisation. Selon les frères Cisterciens, la diffusion sur le Web est un facteur important pour accroître la sensibilisation et la transmission, même si elle ne peut être suffisante. L'accessibilité large et très facile des données sur le site est un avantage pour la transmission, car les pratiques sont documentées avec précision, par des textes riches et par des médias audiovisuels qui fournissent des témoignages précieux pour la transmission, ce que le texte à lui seul ne peut pas offrir. Dans ce sens, ils souhaitent mettre en ligne les vidéos produites par l'IPIR sur la chaîne Youtube de la communauté ${ }^{73}$, ce qui a déjà été fait pour certains ${ }^{74}$.

\section{IPIR à l'international}

Pour terminer ce retour sur l'IPIR, nous avons fait un recensement des chercheurs et spécialistes qui le citaient dans leurs publications à l'étranger afin de mesurer son impact au niveau international. Cécile Duvelle, chef de la Section du patrimoine immatériel de l'UnESCO, a salué « toutes les initiatives [que le Québec] prend, sans attendre, et qui sont vraiment [selon elle], un excellent modèle à suivre ${ }^{75}$ ». L'inventaire multimédia du patrimoine immatériel IPIR

70. Entrevue avec le père Raphaël, de l'abbaye cistercienne de Rougemont, le 10 décembre 2013.

71. Entrevue avec sœur Germaine Julien, des sœurs de la Charité d'Ottawa, le 17 décembre 2013.

72. Entrevue avec le père Raphaël, de l'abbaye cistercienne de Rougemont, le 10 décembre 2013.

73. Ibid.

74. Voir, pourl'Oratoire Saint-Joseph du Mont-Royal, www.youtube.com/watch?v=ItsRmSl1Ktc.

75. Intervention lors du colloque Les mesures de sauvegarde du PCI, Québec 15 avril 2011, citée par Karine Laviolette, « Le Patrimoine immatériel dans la loi sur le patrimoine culturel du Québec », 
est un modèle reconnu sur le plan national et international et constitue un inventaire pionnier. Comme le souligne Karine Laviolette, conseillère en patrimoine immatériel au MCCQ, ces réalisations d'inventaires en partenariat avec le Ministère ont « contribué à faire du Québec un leader dans le domaine du patrimoine immatériel ${ }^{76}{ } \gg$ et plus particulièrement dans le domaine des inventaires du patrimoine immatériel : «Le MCCF a participé à la conception et à la mise en œuvre de l'Inventaire (IREPI) en partenariat avec la Chaire de recherche du Canada en patrimoine ethnologique. Grâce à cette initiative, le Québec a développé une expertise reconnue et a acquis un réel leadership en matière d'inventaire du patrimoine immatériel ${ }^{77}$ »).

Les fondements méthodologiques et techniques sur lesquels repose l'inventaire IPIR ont été élaborés par la Chaire de recherche du Canada en patrimoine ethnologique en partenariat avec le Mcc et la Société québécoise d'ethnologie ( $\mathrm{SQE})$ au fil de nombreuses années, et en premier lieu dans l'inventaire IREPI, premier inventaire du patrimoine immatériel réalisé par la Chaire. Cette méthodologie a connu un succès tel que le titulaire de la Chaire a été sollicité à de très nombreuses reprises, chaque année jusqu'à ce jour, pour partager son expertise dans un domaine encore très peu connu et exploré, tant auprès des chercheurs en sciences humaines et sociales, d'institutions culturelles et d'associations que de gouvernements étrangers et d'universités américaines prestigieuses ${ }^{78}$.

Par exemple, la Mission ethnologie du ministère de la Culture de France s'est inspirée de l'inventaire comme modèle pour réaliser l'inventaire du patrimoine immatériel de la France. De nombreuses collaborations ont été menées durant plusieurs années avec la Direction de l'architecture et du patrimoine du ministère de la Culture de la France suite à l'adhésion de la France en 2006 à la Convention de 1'UNESCO de 200379. D'autres activités scientifiques ont été organisées durant plusieurs années dans le cadre de cette

dans Marie Cornu, Jérôme Fromageau, Christian Hottin (dir.), Droit et patrimoine culturel immatériel », Paris, L'Harmattan, 2013, p. 53.

76. Karine Laviolette, op. cit., p. 53.

77. Karine Laviolette, « Québec ouvre la danse », Continuité, n 127, 2010, p. 47-48.

78. Par exemple, il a occupé la William Lyon Mackenzie King Chair et y a animé entre autres un séminaire sur les politiques et les pratiques du patrimoine immatériel au trimestre d'hiver 2006, puis il a été invité au Media Lab du Miт (Massachussets Institute of Technology) pour développer des recherches sur les narrations transmédia en 2013-2014.

79. Le ministère de la Culture de la France a mandaté la chargée de mission pour le patrimoine ethnologique de la Mission ethnologie, Sylvie Grenet, pour effectuer un stage d'un mois en 2008 au laboratoire de la Chaire à l'Université Laval à cette fin, et a obtenu une subvention dans le cadre de la coopération culturelle France-Québec. Elle a également participé au colloque international de l'Afs (American Folklore Society) organisé à Québec par Laurier Turgeon qui a réuni près de 700 participants sur le thème " Patrimoine immatériel : problématiques, enjeux et perspectives » et a profité des échanges pour nourrir la réflexion sur la réalisation des inventaires. Un membre de l'équipe de la Chaire a réalisé un projet pilote d'inventaire durant l'année 2009 pour envisager les modalités d'application de la méthodologie de cet inventaire au terrain français, plus particulièrement sur le territoire de la ville de Paris. 
collaboration ${ }^{80}$. L'inventaire du patrimoine immatériel de la France a ainsi été réalisé en prenant appui sur l'inventaire réalisé par la Chaire : "Les outils techniques (la fiche d'inventaire), les principes à la base de la démarche de recherche (recherche-action avec restitution des données collectées par le moyen d'expositions, conférences, articles dans la presse locale), et la stratégie de diffusion (sur internet) ont été élaborées sur le modèle de l'Inventaire des ressources du patrimoine immatériel développé au Québec ${ }^{81}$ ». Selon Sylvie Grenet, responsable de l'inventaire du patrimoine immatériel au ministère de la Culture de la France, les catégories du patrimoine immatériel et l'usage du multimédia (texte, photo, audio et vidéo) utilisés pour les inventaires du Québec par la Chaire ont aussi été « source d'inspiration ${ }^{82}$ " pour la France.

Un autre aspect méthodologique distinctif de l'inventaire IPIR qui a servi de modèle est la recherche-action qui garantit l'engagement des communautés dans le processus d'inventorisation et les conduit à se réapproprier les pratiques par des stratégies directes de mise en valeur. Selon Chiara Bortolotto, l'inventaire « développé à Québec [...] a été choisi comme un modèle à la fois pour ses outils techniques, tel que le formulaire d'inventaire, et pour ses principes moraux : tourné vers le public, diffusion internet, recherche-action, soit une recherche qui vise à restituer à ses "propriétaires" les données collectées après avoir été étudiées et organisées par le chercheur. Cette restitution se fait sous forme d'expositions, de conférences, d'articles dans les journaux etc. ${ }^{83}$ » La participation des communautés est l'un des traits caractéristiques

80. Citons-en quelques-unes: exposé sur les inventaires en février 2007 au séminaire du LAHIC à l'École des hautes études en sciences sociales (ÉHÉss, Paris) consacré au patrimoine immatériel, en collaboration avec le département du pilotage de la recherche et de la politique scientifique (Direction générale des patrimoines, ministère de la Culture) pour accompagner les réflexions en cours à la Direction de l'architecture et du patrimoine ; communication avec Daniel Lauzon, du Mcc du Québec, au Service de l'Inventaire du ministère de la Culture de la France, intitulée « Faire l'inventaire du patrimoine immatériel du Québec : problématiques, méthodes et enjeux »; présentation à un colloque, en novembre 2007, « Le patrimoine culturel immatériel de l'Europe : inventer son inventaire », à l'Institut national du patrimoine à Paris (INP) ; organisation de journées d'étude au Musée du Quai Branly en janvier 2010 ; participation aux journées d'étude en juillet 2010 : « Inventorier pour quel patrimoine ethnologique, l'inventaire du PCI au ministère de la Culture ", à l'Institut national d'histoire de l'art de Paris (INHA), organisée par la Mission ethnologie et le LAHIC.

81. Chiara Bortolotto, «Le Transfert d'un standard international », dans Julien Bondaz, Florence Graezer-Bideau, Cyril Isnart, Anaïs Leblon (dir.), Les Vocabulaires locaux du "patrimoine ». Traductions, négociations et transformations, Zurich, Verlag, 2014, p. 112-113. Voir aussi : Sylvie Grenet, « Les Inventaires en France », Culture et recherche, n 116-117, printemps-été 2008, p. $27-28$.

82. Sylvie Grenet, «Les Inventaires en France », dans Christian Hottin (dir.), Le Patrimoine culturel immatériel : premières expériences en France, Paris, Babel, Internationale de l'imaginaireNouvelle série, $\mathrm{n}^{\circ} 25,2011$, p. 77-92.

83. Chiara Bortolotto, «The French inventory of intangible cultural heritage : Domesticating a global paradigm into French heritage regime », dans Regina Bendix et al., op. cit., p. 271 : «IREPI developed in Quebec [...] was chosen as a model for both its technical tools, such as the inventory form, and for its main moral principles : turn to the public, internet diffusion, action research, meaning research that aims to return to their « owners » the collected data after they have been studied and organized by 
de l'inventaire, selon Christian Hottin, conservateur du patrimoine et adjoint au chef du département du pilotage de la recherche et de la politique scientifique au ministère de la Culture de France : «Inspiration has been drawn from the Inventory (Inventaire des ressources ethnologiques du patrimoine immatériel) which was initiated by the Ministry of Culture in Québec and led by Professor Laurier Turgeon of Laval University. The main principle of this inventory is to be elaborated with the active support and participation of all the communities involved ${ }^{84}$. »

Le travail mené dans l'Inventaire IPIR est également un modèle cité par l'historienne Florence Descamps, maître de conférences à l'École pratique des hautes études de Paris : " La France pourrait utilement s'inspirer des travaux menés par le ministère de la Culture du Québec qui depuis quelques années, grâce à la vitalité de certaines associations culturelles, mène une réflexion innovante sur le patrimoine religieux et ses formes tant matérielles qu'immatérielles. On se reportera en particulier au Rapport présenté au Ministère de la Culture par Laurier Turgeon, titulaire de la Chaire de recherche sur le patrimoine ethnologique de l'Université de Laval et par Louise Saint-Pierre, coordinatrice du Projet pilote de l'Inventaire du Patrimoine immatériel religieux dans la même université, Rapport inédit, mai 2008, 96 pages $^{85}$. » Florence Deschamps poursuit en expliquant les raisons historiques de cette avance du Québec : « Ce n'est pas un hasard si le Québec manifeste une telle avance dans la réflexion sur le recueil oral des croyances et des pratiques religieuses ; on se rappelle que le Canada, et plus particulièrement le Québec et l'Université de Laval, ont été pionniers en histoire orale après la Seconde Guerre mondiale et que l'école d'histoire orale québécoise reste actuellement une des plus dynamiques au monde ${ }^{86} . »$ L'IPIR est également cité en exemple par les chercheuses italiennes Maria Teresa Artese et Isabella Gagliardi dans un article récent consacré à l'archivage du patrimoine culturel immatériel ${ }^{87}$. Soulignons enfin le travail de la Péruvienne Valérie Legrand-Galarza, qui s'est inspirée des inventaires de la Chaire pour la réalisation du portail audiovisuel PCIA « Patrimoine culturel immatériel

the researcher. This restitution is made in the form of the exhibitions, conferences, articles in local newspapers, etcetera. »

84. Christian Hottin et Sylvie Grenet, « Reflections on the implementation of the UnESCO 2003 Convention on IcH », dans Michelle L. Stefano, Peter Davis, Gerard Corsane (dir.), Safeguarding Intangible Cultural Heritage, Newcastle University, Boydell Press, 2012, p. 102.

85. Florence Descamps, « Mémoire religieuse, patrimoine immatériel du religieux. Pour la constitution d'archives orales de la foi catholique au $\mathrm{xx}^{\mathrm{e}}$ siècle », In Situ, revue des patrimoines [En ligne], 11 | 2009, mis en ligne le 22 juillet 2009, consulté le 15 avril 2014. URL : insitu.revues.org/4565 ; DOI : $10.4000 /$ insitu. 4565

86. Ibid.

87. Maria Teresa Artese et Isabella Gagliardi, « Cataloging Intangible Cultural Heritage on the Web », in Marinos Ioannides, Dieter Fritsch et Johanna Leissner, Progress in Cultural Heritage Preservation, Berlin, Springer-Verlag, 2012, p. 678. 
des populations andines » du Pérou et de la Bolivie, tant pour le traitement que la diffusion des données ${ }^{88}$.

\section{Conclusion}

Comme l'avait souligné Marius Barbeau il y a plus de 70 ans, les communautés religieuses détiennent une part importante du patrimoine immatériel du Québec. Ces communautés sont aujourd'hui vieillissantes et plus que jamais menacées de disparition. L'approche novatrice d'inventorisation développée dans le cadre du projet IPIR a fait ses preuves ; il est considéré comme un outil efficace de sauvegarde et ses retombées sont fortes tant pour les communautés religieuses que pour l'ensemble de la société québécoise. En dépit de l'important travail accompli, il reste encore beaucoup à faire. L'IPIR n'a recensé le patrimoine immatériel que d'une cinquantaine de communautés religieuses sur un total de plus de 450 au Québec, sans compter les paroisses qui sont au nombre de plus de 1700 . Nous avons privilégié les communautés les plus anciennes et les plus menacées. Ainsi, l'IPIR ne couvre que très peu de communautés catholiques contemporaines (fondées au XIX et au XX ${ }^{\mathrm{e}}$ siècles) et de communautés culturelles (portugaises, italiennes, grecques, etc.), et aucune communauté inuit. Ce travail d'inventorisation est d'autant plus pertinent que depuis octobre 2012 le Québec a adopté une nouvelle loi sur le patrimoine qui inclut pour la première fois dans son histoire le patrimoine immatériel et que l'article 8 de la loi stipule que « Le ministre contribue à la connaissance du patrimoine culturel notamment par la réalisation d'inventaires ». De plus, la sensibilité pour le patrimoine religieux, surtout immatériel, se développe, s'exprimant par l'augmentation du nombre de musées religieux ${ }^{89}$, par la multiplication des colloques et des publications ${ }^{90}$, et aussi par le biais de la demande touristique qui est le reflet - il ne faut pas l'oublier - de la demande sociale. Le tourisme religieux est en plein essor au Québec et ailleurs dans le monde ${ }^{91}$. Il s'agit d'un tourisme dit de niche, c'est-à-dire ciblé, dont la croissance est estimée à $20 \%$ au cours des deux prochaines décennies selon l'Organisation mondiale du tourisme (Омт ${ }^{92}$. Selon les résultats d'une étude

88. Valérie Legrand-Galarza, «Une archive sur le patrimoine immatériel des populations andines au Pérou et en Bolivie », dans Peter Stockinger (dir.), Nouveaux usages des archives audiovisuelles numériques, Paris, Hermès/Lavoisier, 2011, p. 88.

89. Toutes les communautés religieuses importantes possèdent aujourd'hui un musée ; il y en a non moins de 32 dans la province.

90. Il y a eu dix colloques internationaux sur le patrimoine religieux organisés au Québec au cours des dix dernières années, soit un par année en moyenne.

91. Stéphanie Saucier, "Le Tourisme religieux s'accroît ", Le Journal de Montréal, mardi 16 novembre 2010, suite au colloque sur le tourisme religieux au Palais des congrès du 13 au 15 novembre 2010.

92. Stéphane Parent, "Les Québécois pourraient se spécialiser dans le tourisme religieux », Radio-Canada International, 2 juillet 2014 : www.rcinet.ca/fr/2014/07/02/les-quebecois-pourraient-sespecialiser-dans-le-tourisme-religieux. 
récente menée par la Chaire de tourisme Transat de l'UQÀM, ce sont surtout les éléments du patrimoine immatériel religieux (pèlerinages, fêtes, processions, dévotions, chants, musiques) qui retiennent l'attention des touristes ${ }^{93}$. Si la pratique religieuse décline de manière marquée et que les églises se vident au Québec - tant chez les catholiques que chez les protestants -, l'intérêt pour le religieux en tant que patrimoine ne cesse de croître. Plus que de simplement s'intéresser à ce patrimoine, les Québécois semblent vouloir construire une nouvelle relation au religieux par le biais du patrimoine, à savoir instituer de nouvelles formes de religiosité - en dehors du rituel religieux classique - sous le signe de la patrimonialité.

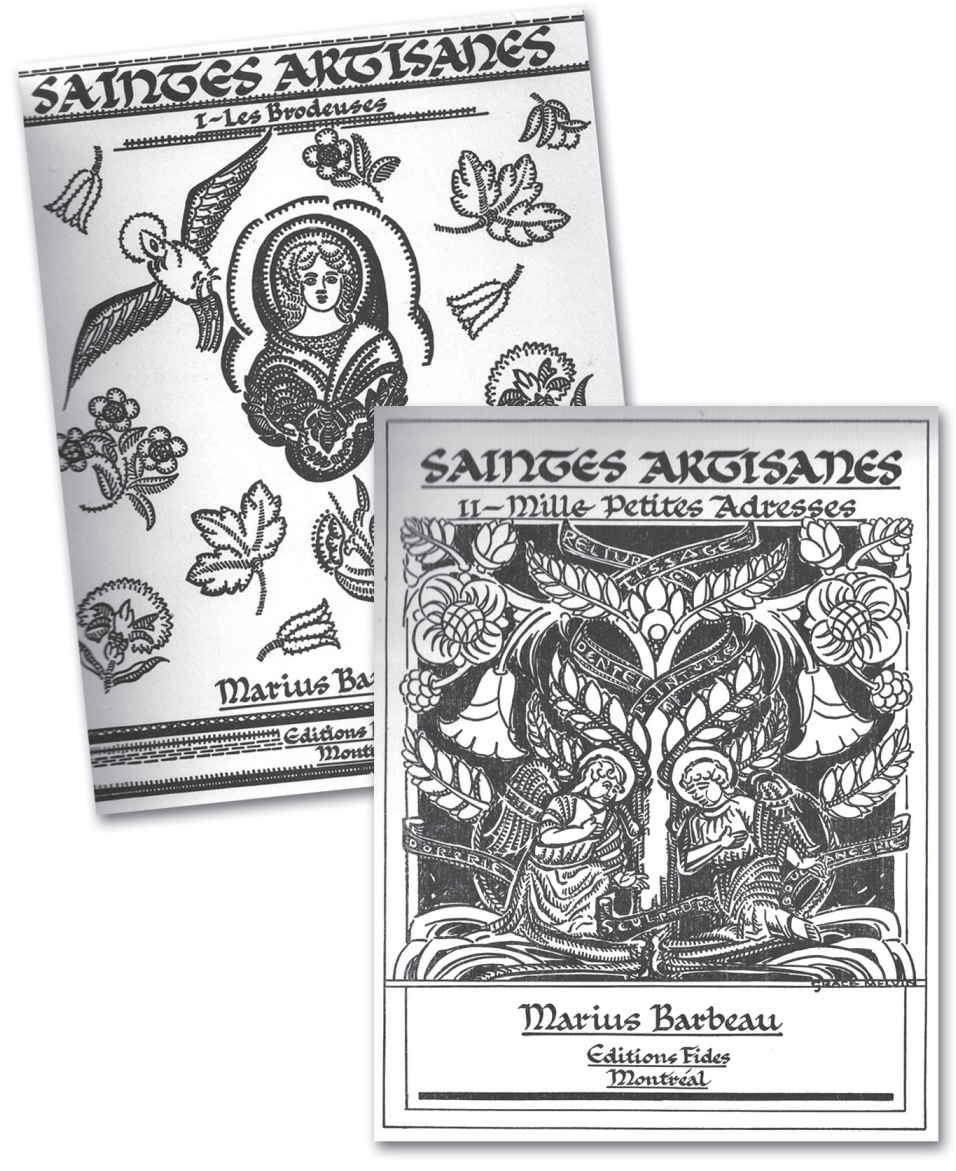

Marius Barbeau, Saintes Artisanes :

I. Les brodeuses et II. Mille petites adresses

(Montréal, Fides, 1943 et 1944).

93. « Le Tourisme religieux : portrait, profil du voyageur et potentiel de développement », Le Réseau de veille en tourisme, Chaire de tourisme Transat, ÉsG-UQÀM, mars 2011. 